\title{
MAZACORNET: Mobility Aware Zone based Ant Colony Optimization Routing for VANET
}

\author{
by \\ Himani Rana \\ A thesis submitted to \\ The Faculty of Graduate Studies of \\ The University of Manitoba \\ in partial fulfillment of the requirements \\ of the degree of \\ Master of Science \\ Department of Computer Science \\ The University of Manitoba \\ Winnipeg, Manitoba, Canada \\ October 2012 \\ (C) Copyright 2012 by Himani Rana
}




\title{
MAZACORNET: Mobility Aware Zone based Ant Colony Optimization Routing for VANET
}

\begin{abstract}
Vehicular Ad hoc Networks (VANET) exhibit highly dynamic behavior with high mobility and random network topologies. The performance of Transmission Control Protocols in such wireless ad hoc networks is plagued by a number of problems: frequent link failures, scalability, multi-hop data transmission and data loss. To address these VANET routing issues, I have used the ideas from swarm intelligence.

The Ant Colony Optimization (ACO), which is a branch of swarm intelligence, is the main source of my inspiration. I have designed an ant-based routing algorithm which addresses routing issues prevalent in VANETs: adaptivity, robustness and scalability. One attractive feature of ACO is that they provide multiple routes from source to destination, resulting in more robust network. In this work, together with ACO, I have used the ideas from zone routing protocols to develop my algorithm: Mobility Aware Zone based Ant Colony Optimization Routing for VANET that exhibits locality and scalability.
\end{abstract}




\section{Acknowledgments}

I would like to begin by thanking almighty God for giving me the strength to carry out this research.

I want to thank my two supervisors, Dr. Parimala Thulasiraman and Dr. Ruppa Thulasiram, who has made sure that I most certainly carry my M.Sc. thesis project all the way through to the defense in this uncertain world. They constantly communicated with me through emails, and finding time in their busy schedules to meet with me at moment's notice. They have my utmost gratitude.

I would also like to thank the members of my thesis examination committee- Dr. Pourang Irani and Dr. Pradeepa Yahampath — for giving me valuable comments and suggestions on my thesis.

Finally, I would like to thank the four pillars of my life: my father, husband, mother and sister. Their emotional and moral support helped me to achieve my goals. They told me to reach for the stars. I think I got my first one. We made it. 
This thesis is dedicated to the person who never stop believing in me and who along with god, have been my 'footprints in the sand' my father Dr. Vijay Kumar Rana. 


\section{Contents}

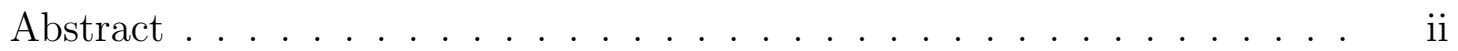

Acknowledgments . . . . . . . . . . . . . . . . . . iii

Dedication. ............................. iv

Table of Contents . . . . . . . . . . . . . . . . . . . . vi

List of Tables . . . . . . . . . . . . . . . . . . . . vii

List of Figures . . . . . . . . . . . . . . . . . . . viii

1 Introduction 1

1.1 Goal of thesis $\ldots \ldots \ldots \ldots \ldots \ldots$

1.2 Thesis Organization $\ldots \ldots \ldots \ldots \ldots \ldots \ldots$

2 Vehicular Ad hoc Network 5

2.1 Introduction to Vehicular Ad hoc network $\ldots \ldots \ldots \ldots$

2.1 .1 Challenges of VANET $\ldots \ldots \ldots \ldots$. . . . . . . . . . 7

2.2 Radio Propagation models . . . . . . . . . . . . . . . . . . . . . . . 10

2.2 .1 Free Space Radio Propagation . . . . . . . . . . . . . . . . . 10

2.2 .2 Two Ray Ground Radio Propagation . . . . . . . . . . . . . 10

2.2 .3 Ricean and Rayleigh Fading Models. . . . . . . . . . . . . . . 11

2.2 .4 Nakagami Radio Propagation . . . . . . . . . . . . . . . . 11

2.3 Mobility models . . . . . . . . . . . . . . . . . . . . . . . 12

2.3 .1 Macro-mobility models . . . . . . . . . . . . . . . . . . . 12

2.3 .2 Micro-mobility models $\ldots \ldots \ldots$

2.4 VANET Routing Algorithms . . . . . . . . . . . . . . . . . . . . 14

2.4 .1 Routing protocol based on routing strategies . . . . . . . . . 14

Topology Based Routing . . . . . . . . . . . . . . . . . . 14

Position Based Routing. . . . . . . . . . . . . . . . . . 15

Cluster Based Routing . . . . . . . . . . . . . . . . . . 19

Broadcast Routing . . . . . . . . . . . . . . . . . 20

Geocast Routing . . . . . . . . . . . . . . . . . . . . . . . . 21

2.4 .2 Routing protocol based on constructed path . . . . . . . . 22

2.5 Summary $\ldots \ldots \ldots \ldots \ldots \ldots \ldots$ 
3 Ant Colony Optimization 24

3.1 Ant Behavior . . . . . . . . . . . . . . . . . . . . . 24

3.2 Ant Based Algorithms . . . . . . . . . . . . . . . . . . . 25

3.2.1 Ant Based Algorithms for Ad hoc network . . . . . . . . . . . 25

3.2.2 Ant Based algorithms for VANET . . . . . . . . . . . . . . . . 29

3.3 Summary . . . . . . . . . . . . . . . . . . . . 30

4 An Improved Mobility Aware Zone based Ant Colony Optimization Routing for VANET $\quad 32$

4.1 Link Stability . . . . . . . . . . . . . . . . . . . . . . . . . . . . 32

4.2 Ant colony-based Algorithm for VANET . . . . . . . . . . . . . . . . 34

4.3 Zone-based ant colony VANET algorithm . . . . . . . . . . . . . . . . 35

Route discovery within the zone . . . . . . . . . . . . . . . 38

Route discovery between the zones . . . . . . . . . . . . . . . 38

Route maintenance . . . . . . . . . . . . . . . . . 38

4.4 Summary $\ldots \ldots \ldots \ldots$. . . . . . . . . . . . . . . . . . . . . . 39

5 Simulation Results $\quad 40$

5.1 Simulation environment . . . . . . . . . . . . . . . . . . . . . 40

5.2 Simulation Process $\ldots \ldots \ldots$. . . . . . . . . . . . . 41

5.3 Simulation Setup . . . . . . . . . . . . . . . . . . . . . . . . . . . . . . . . . . . . . . . . . . .

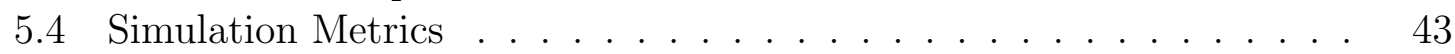

5.5 Simulation Results . . . . . . . . . . . . . . . . . . . . 44

5.6 Summary . . . . . . . . . . . . . . . . . . . 51

\begin{tabular}{lll}
\hline 6 & Conclusion & 52
\end{tabular}

\begin{tabular}{lll}
\hline 7 & Future Work & 54
\end{tabular}

\begin{tabular}{ll}
\hline Bibliography & 55
\end{tabular} 


\section{List of Tables}

2.1 Compare Vehicular Traffic Tools [FHFB07]-[MCCM08] . . . . . . . . 13

$3.1 \quad$ Ant based routing algorithms with proposed year [SBHR96][KO07] . 31 


\section{List of Figures}

2.1 Vehicular Ad hoc Network . . . . . . . . . . . . . . . . . . . 7

$2.2 \quad$ DSRC Spectrum distribution $\ldots \ldots \ldots \ldots$

3.1 Ants behavior . . . . . . . . . . . . . . . . . . . 25

4.1 MAZACORNET example . . . . . . . . . . . . . . . . . . 36

$5.1 \quad$ VANET Simulation Process $\ldots \ldots \ldots \ldots$. . . . . . . . . . . 42

5.2 End to End Delay: MAZACORNET vs Other routing algorithms . . 45

$\begin{array}{lll}5.3 & \text { Packet Delivery Ratio: MAZACORNET vs Other routing algorithms } & 46\end{array}$

5.4 Routing Overhead: MAZACORNET vs Other routing algorithms . . 47

5.5 Throughput: MAZACORNET vs Other routing algorithms . . . . . . . 48

5.6 Packet delivery ratio with various zone radius $\ldots \ldots \ldots \ldots$

5.7 End to end delay with various zone radius $\ldots \ldots \ldots \ldots$

5.8 Routing overhead with various zone radius $\ldots \ldots \ldots \ldots$ 


\section{Chapter 1}

\section{Introduction}

Vehicular Ad-hoc Network (VANET) is a subclass of an ad hoc network. Vehicles in VANET travel at high speeds and communicate between themselves through a wireless network or centrally through road side units. The communication between two vehicles is called a vehicle-to-vehicle (V2V) communication, whereas, communication between the vehicle and the road side unit is known as vehicle-to-infrastructure (V2I) communication. In this work, I consider V2V communication.

In $\mathrm{V} 2 \mathrm{~V}$, each vehicle (a vehicle in a network) transmits a message to other vehicles using on-board units. However, the movement of vehicles although are in an organized fashion, that is, in accordance to traffic rules, traffic signals, signs and speed signs, pose some challenges for VANETs [BEH04]. First, network congestion occurs very frequently, making it difficult for packets to reach their destination. Second, the vehicle connectivity changes rapidly due to mobility of vehicles affecting the lifetime

of links established between the vehicles causing frequent disconnection of many paths before they can be successfully used. Therefore, vehicles connectivity depends on 
the vehicles density and mobility patterns. Vehicles use wireless network as the primary medium to communicate with other vehicles in their radio range using routing protocols.

VANET, as in other wireless networks, uses routing protocols to exchange topology related information within the network. Once the information is exchanged, the routing algorithm computes the most optimal path between the vehicles. Several VANET routing algorithms exist in the literature, but most of these algorithms discover a single path between source and destination which do not fully use route discovery information. As discussed above, mobility of vehicles lead to link failure causing network delay and overhead. Thus, to overcome such issues I have designed an algorithm based on the principles of swarm intelligence.

Bio-inspired or nature-inspired algorithms have shown [DCG05, OTT08, WOTT09] to provide efficient routes or solutions for ad hoc networks. These algorithms have a number of benefits compared to other routing algorithms [CJ03, [PR99]. For example, they reduce the routing overhead by sharing local information for future routing decisions. They also provide multiple paths enabling selection of another route in case of link failure on the previously selected path. The idea of nature-inspired algorithms is derived from the behavior of insects such as bees, ants or termites. One of the nature inspired algorithms that is well studied in ad hoc networks is the Ant Colony Optimization (ACO) CD98]. I have used the ACO concept to design my routing algorithm which creates multiple transmission paths between source and destination vehicles to efficiently enhance network bandwidth and fairly utilize network resources.

Also, to my knowledge, Mobility-aware ant colony optimization routing algorithm 
for vehicular ad hoc networks (MAR-DYMO) [CCC11] is the only nature inspired research work done on VANET. This protocol sends a packet to all other vehicles in the network using broadcast mechanism consuming large amount of bandwidth. To make effective bandwidth consumption I have developed a hybrid algorithm that uses the concept of zones. A network is subdivided into zones with each vehicle belonging to one or two overlapping zones. I have used proactive approach to find a route within a zone and reactive approach to find routes between zones using the local information stored in each zone thereby trying to reduce broadcasting and congestion.

\subsection{Goal of thesis}

I have designed a hybrid, multipath swarm-based routing algorithm that maintains a network proactively within zones and reactively find routes between the zones. My algorithm adapts to the dynamics of VANET, and is scalable and robust to link failures. To evaluate my algorithm, I have used three different tools. I have used VanetMobiSim traffic simulation tool to generate realistic vehicular mobility traces, NS2 network simulator, which generates the communication environment and supports simulation of routing, multicast, transport protocol over wired and wireless network and Awk to provide interpret the NS2 file. Finally, after running simulations and obtaining the results of my algorithm I have compared my algorithm results to the existing protocols: AODV, GPSR, AMODV and evaluated the performance of my algorithm. 


\subsection{Thesis Organization}

Apart from presenting the concepts of vehicular ad hoc networks, Chapter 2 explains the wireless access for the vehicular network and routing protocols present for the vehicular ad hoc network. Chapter 3 explains the Ant colony optimization and applications of ACO in the networking and other fields. Chapter 4 introduces my proposed routing algorithm for VANET which is scalable and promises fault tolerant communication in such a dynamic environment. Chapter 5 provides simulation results describing (a) the advantages of using the proposed routing algorithm (b) comparing my algorithm with other existing algorithms. Finally, Chapter 6 presents a summary of this thesis and Chapter 7 suggests some possible future work. 


\section{Chapter 2}

\section{Vehicular Ad hoc Network}

In this chapter, I first explain VANET and few challenges in this area. Then, I discuss regarding the propagation models and about the mobility models for VANET. I will also provide the information regarding the routing algorithms present for the VANET.

Amaya and Magee AM08, have quantified the progress of wireless technology. Since the introduction of the cellular technology, the progress has followed annual increases greater than $50 \%$. They predicted wireless interfaces to be the dominant way of connecting to the Internet. Nowadays, we can see that mobile communication is applied to almost all the aspects of business and personal computing [Lab08]. These mobile units can communicate in two ways: infrastructured networks and infrastructureless networks.

In infrastructured networks all the nodes are connected to a central node

which acts as a communication agent between the vehicles. Examples of this kind of network includes GSM [MP92] and WLAN [BCG01]. Infrastructureless networks 
also known as wireless ad hoc network has no physical connections between nodes. Due to the mobility of the nodes, ad hoc networks have no fixed topology. All the routing decisions are made dynamically. VANET is an example of such a network, where designing a network protocol is complex because of a dynamic environment.

In the next section, I will present more detailed description of VANET along with its features and challenges.

\subsection{Introduction to Vehicular Ad hoc network}

VANET is a special kind of ad hoc network, where each vehicle acts as a node and communicates with nearby vehicles and roadside units, which are mounted in centralized locations like intersections and parking. Figure 2.1 shows different ways of communication that can exist on the road.

Vehicle to Vehicle Communication (V2V): In V2V communication nearby vehicles exchange data by the use of short range wireless technologies, Wi-Fi and WAVE [XSYA12]. Vehicles have a special electronic device that allows them to receive or relay messages.

Vehicle to Infrastructure Communication (V2I): In V2I vehicles are connected to the nearby road infrastructure via continuous wireless communication i.e. through Wi-Fi hotspots or long/wide range wireless technologies for exchanging information relevant to the specific road segment. 
Figure 2.1: Vehicular Ad hoc Network

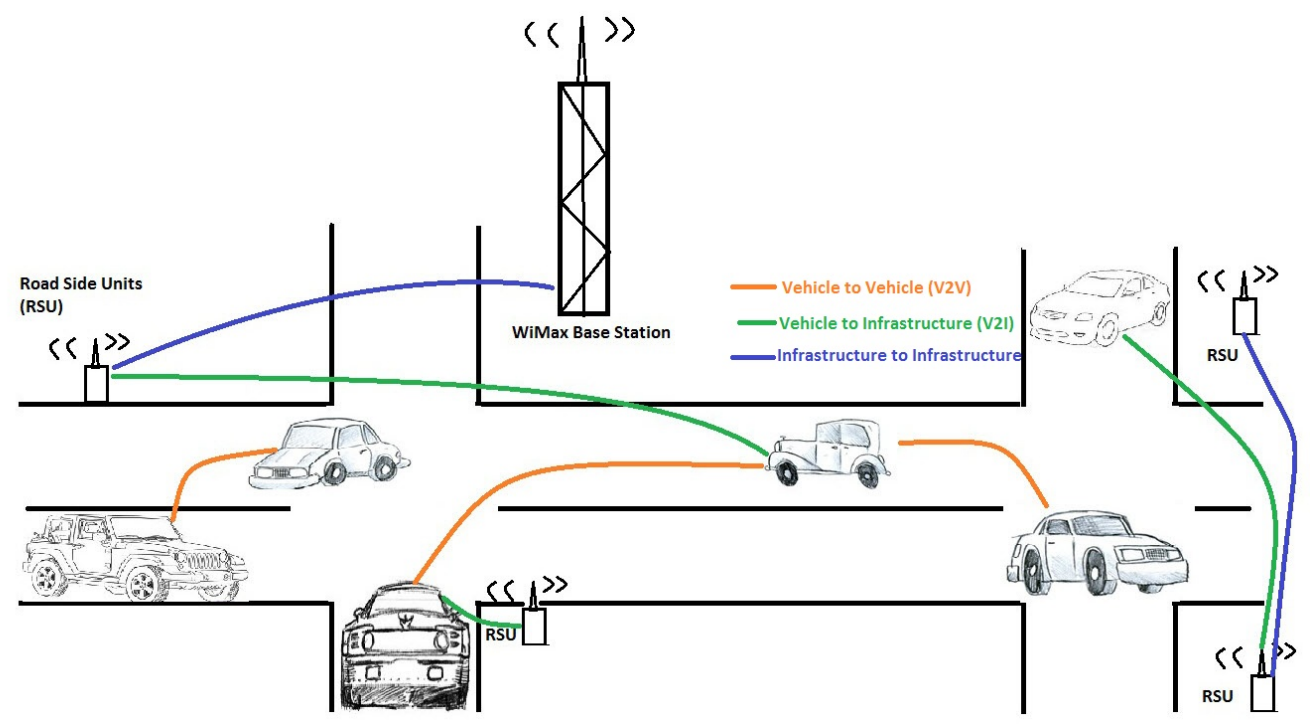

VANET provides safety and comfort to the passengers. Vehicles can share realtime information to avoid collisions and other road hazards as well as coordinate driving systems. However, the movement of vehicles in an organized fashion according to traffic rules, traffic signals, signs and speed signs pose many challenges for VANETs. In the next section I will briefly outline some of the challenges and research in VANET.

\subsubsection{Challenges of VANET}

Dynamic network topology: In vehicular network the life time of the links are affected by the mobility of the vehicles i.e. the connectivity between the vehicles. Therefore, the vehicular routing algorithms should consider the mobility patterns along with the propagation and traffic conditions.

Routing: Due to dynamic network topology the routing tables of each vehicle needs to be configured requiring lots of communication and causing congestion XL08, 
KSLB11]. Fragmentation of the network occurs very frequently, in which chunks of the network are unable to reach the destination. Therefore, routing algorithm should be capable of transmitting a data using different route in case of congestion.

Security: is another issue studied in VANET [SASS10]. Due to the non-existence of infrastructure that provides security services centralization like user authentication or packet ciphering, there are many chances of having network attacks. The issue to be addressed includes trust, resiliency, efficiency and privacy.

Quality of Service (QoS): To achieve QOS in wired network there are several resource reservation mechanisms but because of high mobility and high density of vehicles in VANET it is hard to execute such mechanisms. There has been some work on achieving QOS in VANET [SYK06] [YRB10].

Power Management: In VANET we are not concerned about energy efficiency, but rather the transmission power. When the transmission power is too high, the on-going transmission could disrupt another transmission due to interferences. So for denser network it is advised to use less power. Ongoing research in this area CGMV05, LNM04 discusses several algorithms where power is adjusted to keep nodes within maximum and minimum threshold.

In general, VANET is similar to an ad hoc network. However, due to issues such as speed and line of sight, the standard IEEE 802.11 used in ad hoc network is not well suited for VANET environment. Wireless Access in Vehicular Network (WAVE) whose outstanding component is the IEEE 802.11p amendment [RRS10] is the new standard that is being developed for VANET. Other than IEEE 802.11p it is possible to use IEEE 802.11 b wich was initially designed for low-mobility indoor 
wireless scenarios but now days are being used for VANET communications GSPM08, WWM07]. Following are the main enhancements done on IEEE 802.11 to add support to wireless local area networks (WLANs) in a vehicular environment [YW08]:

- Ranges from 1 meter up to 1000 meters

- Short latency

- Nature of the automotive applications to be supported (e.g. reliable broadcast)

IEEE 802.11p standard is designed to operate in the DSRC band which is different than IEEE 802.11a which operates in 5GHz ISM band. Figure 2.2 shows US distribution of DSRC spectrum. The central one is the Control Channel $(\mathrm{CCH})$ and is used for safety-critical communications only. The first and the last channel are for special uses. Two adjacent service channels may be used as one $20 \mathrm{MHz}$ channel.

Figure 2.2: DSRC Spectrum distribution

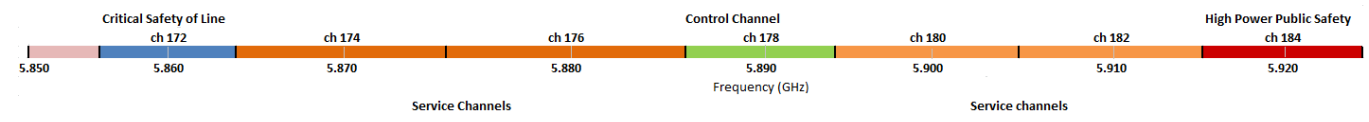

Deploying the IEEE standards for VANET requires high cost and great efforts. Therefore, studies on VANETs are done using simulations. Simulations must consider the possibilities that can occur on real road. This includes mobility models and propagation models which consider issues such as vehicle's movement pattern, vehicle density, vehicle velocity and fading scenarios that significantly affect simulation results. In Section 2.2 and 2.3 propagation and mobility models for VANETs are discussed respectively. 


\subsection{Radio Propagation models}

Propagation is the behavior of radio waves when they are transmitted or propagated from source to the destination. During transmission, waves can be affected by phenomena of reflection, refraction, absorption and scattering. Below is the evolution and details of the radio propagation for fading scenario.

\subsubsection{Free Space Radio Propagation}

This was the earliest model used for Mobile Ad hoc Network (MANET) research. It is the signal propagating through open space with no environmental effects. It uses just Line of Sight (LOS) parameter. If LOS is there between sender and receiver then

only communication takes place. Otherwise, the signal is blocked and no communication takes place Rap01. This model is not suitable for VANET as received power is dependent on the transmitted power, antenna gains and on the distance between the sender and the receiver. Obstacles are not considered.

\subsubsection{Two Ray Ground Radio Propagation}

The two ray ground radio propagation model is highly recommended by MANET researchers. This model is more realistic as along with direct LOS path this model also considers ground reflected propagation path between sender and receiver. This model is not suitable for VANET as obstacles are not considered and sender and receiver have to be on same height Rap01. 


\subsubsection{Ricean and Rayleigh Fading Models}

In Ricean and Rayleigh fading models Rap01, fading is mostly caused by multipath propagation of the radio waves. Rayleigh fading occurs, if there are multiple indirect paths between the sender and the receiver. Whereas, ricean fading occurs, when there is one dominant (line of sight) path and multiple indirect signals.

\subsubsection{Nakagami Radio Propagation}

This probabilistic model describes the rapid fluctuations of the received signal due to multipath fading. This model can form a perfect free space channel, moderate fading channel on highway, and dramatically fading channel in urban areas. In the Nagakami Model with a positive integer value for the fading parameter $m$ the data packet reception probability is given by

$$
P_{r}=\frac{2}{\Gamma(m)}\left(\frac{m}{\Omega}\right)^{m} r^{2 m-1} e^{-\left(\frac{m r^{2}}{\Omega}\right)}
$$

Here, $\Gamma(m)$ is the gamma function, $m$ is the fading parameter defined as $m=\frac{\Omega}{E}\left[\left(r^{2}-\right.\right.$ $\left.\Omega)^{2}\right] \geq \frac{1}{2}$ and $\Omega=E\left(R^{2}\right)$ is used to denote the amplitude of the received signal. The physical layer (i.e. the propagation model chosen) has a high impact on the network layer, where routing protocols and algorithms are used for communication between vehicles. This model is highly applicable for the use in VANET [RRC07]. By varying the fading parameter $m$ we can form highly fading scenario like urban or city as well as free way of highway.

In this thesis, I consider the Nakagami model. The probability distribution func- 
tion is incorporated in my ACO algorithm to study the data packet reception probabilities in dynamic VANET environment.

\subsection{Mobility models}

The pattern which defines the movements of mobile nodes within the simulated area during a simulation time is known as mobility model. Earlier, in case of mobile ad hoc network, mobility models assumed the rectangular simulation area to be without an obstacle which is not realistic in case of VANET. So recent studies talks about different mobility models available for VANET [MPGW06, CB05, SJ04]. Mobility models can be divided into following two categories [CBD02, [FHFB07]: Macro-mobility models and Micro-mobility models.

\subsubsection{Macro-mobility models}

This model focuses on the motion constraints and the vehicular traffic. Motion constraints include streets, crossroads, roads, speed limits, number of lanes, traffic light and the traffic signs description establishing the traffic rules. Vehicular traffic includes road topology, traffic density, traffic flows and initial vehicle distribution. The main macro mobility characteristics used during simulations are:

- Road topology is defined by graphs. Graphs can be user defined, random or extracted from real maps

- Vehicles initial and destination point and the velocity of vehicles

- Number of lanes and traffic lights on the roads 
Table 2.1: Compare Vehicular Traffic Tools [FHFB07]-[MCCM08]

\begin{tabular}{|c|c|c|c|c|c|}
\hline Features & & VanetMobiSim & SUMO & STRAW & Freesim \\
\hline \hline Maps & & & & & \\
\hline & Real & $\mathrm{X}$ & $\mathrm{X}$ & $\mathrm{X}$ & $\mathrm{X}$ \\
\hline & User Defined & $\mathrm{X}$ & $\mathrm{X}$ & & \\
\hline & Random & $\mathrm{X}$ & $\mathrm{X}$ & & \\
\hline Traffic Model & Manahattan & & & & \\
\hline & Multilane roads & $\mathrm{X}$ & $\mathrm{X}$ & $\mathrm{X}$ & \\
\hline & Lane changing & $\mathrm{X}$ & $\mathrm{X}$ & $\mathrm{X}$ & \\
\hline & Overtaking Criteria & $\mathrm{X}$ & & & \\
\hline & Different vehicle type & & $\mathrm{X}$ & & \\
\hline Traces & & & & & \\
\hline & NS-2 trace & $\mathrm{X}$ & & & \\
\hline & NCTUns trace support & & & & \\
\hline & Import different formats & $\mathrm{X}$ & $\mathrm{X}$ & $\mathrm{X}$ & \\
\hline
\end{tabular}

\subsubsection{Micro-mobility models}

This model focuses on the behavior of a driver when interacting with other drivers or with road infrastructure. The behavior is based on the driver's personal aspect like gender, age and even the mood while driving.

Table 2.1 displays few mobility model generator tools along with their characteristics.

From the table we can see VanetMobiSim provides support trace files for NS2 along with many other features. It supports both micro and macro mobility models. Therefore, for my research I have used VanetMobiSim for generating the real world traffic scenarios. 


\subsection{VANET Routing Algorithms}

Before moving on to discussing about the ACO routing for VANET, this section presents the existing routing algorithms in VANET. The classification of routing protocols is based on the routing strategies and the number of path constructed [WW11] as explained in subsections below.

\subsubsection{Routing protocol based on routing strategies}

Based on the routing strategies, routing protocols are categorized into five different groups [JM96]: (1) topology-based routing, (2) position-based routing, (3) cluster-based routing, (4) broadcast routing and (5) geocast routing.

\section{Topology Based Routing}

Topology based protocols, such as AODV and Fish Eye Routing (FSR) [PGC00] can be applied to VANETs. Namboodiri et al. [NAG04] evaluated that locating, updating and maintaining a long route in VANET using AODV is impossible due to the dynamic nature of VANET. Frequent route failures disrupt the communication between the nodes. Although AODV is not sufficient for networks that contain long route paths, it is scalable for small networks where the path length is a few hops long.

FSR is a link state routing protocol where topology map is maintained at each node. Instead of propagating the link state updates to entire network, FSR sends the link state updates only to the immediate neighbors. Different frequencies are used for broadcasting the link state information to the nodes depending on their distance from current node. If the destination node is closer to the current node, the information 
is broadcasted with higher frequencies compared to the distant nodes.

\section{Position Based Routing}

Position based routing can be classified as Non-Delay Tolerant Network (NonDTN), Delay Tolerant Network (DTN) and Hybrid routing. Each is explained below.

Non-Delay Tolerant Network Non-DTN protocols do not consider broken connectivity. The node sends its packet to the neighbor that is closest to the destination. If the node is closer to the destination than any other node, the forwarding strategy fails and we can state that the packet has reached the local maximum at a given node. Several protocols that fall into this category are: Position-Based Routing with Distance Vector (PBR-DV) Wat10], Connectivity Aware Routing (CAR) [Val07], Geographic Source Routing (GSR) [LHT ${ }^{+}$, Landmark Overlays for Urban Vehicular Routing Environment (LOUVRE) [LLHG08] and Greedy Perimeter Stateless Routing (GPSR) Bra00]

PRB-DV uses several approaches, such as greedy routing, reactive routing and position based routing for communication among the nodes. The packet is forwarded towards the destination using the greedy approach but once the packet reaches the local maximum, the AODV (i.e. reactive approach) is used for recovery. The node at local maximum state broadcasts the request packet with node's position and destination location. Upon the receipt of the data packet, the node checks to see if its position is closer to the destination. If it is not closer to the destination, it will rebroadcast the packet and send a reply to the node from which it received the packet. This protocol is not efficient, as flooding is involved in route discovery, which may 
cause network congestion.

In CAR, path discovery is done by using the AODV approach and broadcasting is done by using the PGB approach. A node that forms the path stores the anchor points (i.e. nodes present on road curves or road crossings) in their path discovery packets instead of storing information about the previous node, which forwards the path reply or the previous node, obtained from backward learning. The destination node selects the best path having lower delays and better connectivity among the multiple path discovery packets received by it. These anchor points are used by CAR to send the route reply message to the source. Guards are used for tracking the current position of the destination. Guarding node can either redirect the packet to the destination or add information to the packet so that it reaches destination.

GSR is the combination of position based routing with topological knowledge. Lochert et al. [LHT ${ }^{+}$introduced GSR for city environment, which uses maps and based on the overlay graph, the algorithm computes the shortest path. The vertices's of the graph are considered as junction nodes and the edges are considered as streets which connect those junction nodes. Routes are established and packets are forwarded greedily to the destination using these junction nodes. This protocol is designed for city environment so it ignores the network where there is less nodes i.e. sparse network.

LOUVRE is the density based geographic greedy overlay link state routing protocol. It uses road length and density for route creation. The link state table is constructed using Dijkstra's routing algorithm and stores the information for routing between the overlay nodes. Here, on-board NAV systems provide the map, which is 
used for designing a road topology graph. Vertices's of the graph are considered as the roads and the edges denote the connectivity between the roads. In LOURVE, there are two types of routing: inter-road routing or overlay routing and intra-road routing and underlay routing. Inter-road routing provides the routing direction while the intra-road routing provides guaranteed greedy forwarding. LOURVE has two bootstrapping stages. In the first stage, the nodes do not have any knowledge of roads' density. Classical geographic routing approach is used in this stage for packet routing. In the second stage, initially the nodes will not have any roads' density knowledge but after the first periodic update, nodes will have the knowledge of all the neighboring roads' density. There are chances of a node encountering a local maximum due to the lack of density information. Therefore, two recovery strategies are designed based on the application type. If it is time sensitive application, packets are routed back to the previous road where next best path is identified, and packet is forwarded toward the destination. If the application is delay-tolerant, then carry-forward approach is used. Node stores and carries the packet until it finds another node based on the routing table.

GPSR uses beacon to select the node that is closest to the destination and uses a greedy mode to deliver the packet. Once the packet reaches the local maximum, perimeter mode is used to recover from this state. After recovering from the local maximum state, packet resumes the forwarding process in the greedy mode until the local maximum is encountered. Packet forwarding is done dynamically and the node remembers only one hop neighbor location. 
Delay Tolerant Network In VANET, nodes are very mobile. In the case of disconnections between the nodes, the node stores and carries the packet for some distance, until another node is found to forward the packet. DTN follows the carry and forward strategy. The Vehicular Assisted Data Delivery (VADD) [ZC08] and the Geographical Opportunistic (GeOpps) [LM07] routing are two protocols, that fall under this category.

In VADD, the path with the smallest packet delivery delay is selected as a forwarding path. The expected delivery delay of a path is modeled by the road density, the distance and the vehicle's velocity. The packet carrier selects the best forwarding path by switching among three different packet modes: straight way, destination and intersection. Several variations of VADD protocols were used in the intersection mode. These protocols choose the next forwarding node, once the next forwarding path has been identified. Location First Probe (L-VADD) selects the node closest to the preferred forwarding path, although the selected node is going away from the forwarding path. Routing loops may occur in L-VADD, which can be eliminated by using Direction First Probe (D-VADD). In D-VADD, the nodes that are moving towards the preferred direction are selected first. Among the selected nodes, the node that is closest to the selected direction is chosen as the next hop node. Multiple-Path Direction First Probe (MD-VADD) selects multiple nodes, which are moving towards the forwarding path; as such, the probability of missing the node that offers shorter time to destination is decreased. As multiple nodes are selected for forwarding the packets, more network traffic is generated, causing congestion. Hybrid Probe (HVADD) combines the features of both L-VADD and D-VADD, resulting in no routing 
loops and long packet delays.

GeOpps uses the vehicle Navigational Systems (NS) to route the packet to its destination. The node, which carries the message closer to its destination, is selected based on the routes suggested by the vehicle's NS. The protocol estimates the packet's arrival time to destination and calculates the shortest distance from packet's destination to the nearest point in the vehicle's path. If any other vehicle with shorter estimated arrival time is identified, the packet is transferred to that vehicle. Same process is followed until the packet reaches its destination.

Hybrid Routing Hybrid routing is a combination of both, the non-DTN and the DTN approach. GeoDTN + Nav [CWT $\left.{ }^{+} 08\right]$ is a hybrid protocol that uses the perimeter mode, greedy mode and the DTN mode for communication among the nodes. The connectivity of the network is estimated based on the number of hops the packet traveled, the neighbors delivery quality and the destination direction. Based on these estimates, routing is switched between the non-DTN and the DTN mode. The Virtual Navigation Interface (VNI) is used to identify the delivery quality of neighbors and to provide best-effort routing decisions.

\section{Cluster Based Routing}

Cluster Based Routing (CBR) [LG97] nodes are clustered to create a virtual network infrastructure. The geographic area is divided into a grid. If the node falls within the grid area, it is elected as a cluster head. The cluster head is responsible for inter and intra-cluster coordination. Nodes in the same cluster communicate directly, whereas, nodes in different clusters communicate using the cluster head. The 
source node in the different cluster sends the data packet to its neighboring cluster head directly, which then forwards the packet following the same policy until the packet arrives at the destination. CBR saves memory space by not caching the routing tables and also increases the packet delivery ratio.

\section{Broadcast Routing}

In Broadcast Routing, network flooding is done to send a packet to all the nodes in the network. Flooding technique can be used in small networks, but as the network grows the overall network performance drops. Every node broadcasts messages at the

same time, causing network congestion and high-bandwidth consumption. BROADCOMM [DDB05], Urban Multi-Hop Broadcast (UMB) [KEOUO04] and Vector Based Tracing Detection (V-TRADE) [SFL $\left.{ }^{+} 00\right]$ are few examples of broadcast routing protocols.

BROADCOMM is designed for highway networks, where the highway is divided into virtual cells. Nodes in the highway are organized in a hierarchical fashion. The first level in the hierarchy includes all the nodes in one cell, and second level includes only a few nodes, known as cell reflectors, which are located close to the geographical center of the cell. The cell reflector handles the messages that come from the nodes within the same cell or cells, to the closely located neighboring nodes. Although the routing overhead is reduced and the message broadcasting delay in this protocol is low, it is only feasible to be employed for the highway networks.

UMB was designed to overcome the packet collision and congestion problems. The sender node selects the furthest node and assigns it the packet for forwarding. 
Repeaters are installed at every road intersection to forward packets to all road segments.

V-TRADE is a GPS based broadcasting protocol, which classifies the neighbors into different groups, based on the position and the movement information of the node. The node located at the border of the group is used to re-broadcast the message to the nodes within the group. This protocol improves the bandwidth utilization, as fewer nodes rebroadcast the message.

\section{Geocast Routing}

Geocast Routing is a location based routing, where packets are delivered within a specified geographical region. Geocast routing restricts the network congestion and limits the message overheads by defining the forwarding zone and restricting the flooding within that zone. Briesemeister et al. [BSH00] proposed a geocast scheme for reducing the number of broadcasts to avoid packet collisions. Packet is rebroadcasted based on the waiting time and this waiting time is identified by looking at the distance of the current node from the sender node. At the expiration of waiting time, if the node does not receive the same message from other nodes then it will rebroadcast the message. This approach reduces the broadcast storm and optimizes the packet forwarding. Inter-Nodes Geocast Protocol (IVG) [BB03] is designed to broadcast the alarm message to all the nodes in risk area on highway. Maihofer et al. [MLS05] designed an abiding geocast approach where packets are delivered to all the nodes which are inside the geocast destination region. Abiding geocast proved to be useful for application like publish and subscribe and position based advertising. 


\subsubsection{Routing protocol based on constructed path}

Based on the number of constructed paths, there are: (1) single path (2) carryand-forward path and (3) multipath routing protocols with pros and cons in each of them.

In single path routing the new route discovery that is required during the link failure adds to delay and network overhead. The carry-and-forward routing approach, where nodes carry a packet to the new receiver that moves into its vicinity, is only applicable for delay tolerant applications. On the other hand, multipath routing provides an alternate route upon failure of the primary route. This provides fault tolerance and lowers the routing overhead because of fewer route discovery operations. Few existing multipath routing algorithms include Ad hoc On-Demand Multi-path Distance Vector (AOMDV) [MD01], S-AOMDV [CXJJ09], AODVM [YKT03. Since my proposed algorithm is for multipath routing, I briefly describe the different multipath routing algorithms existing in the literature.

\section{Ad hoc On-Demand Multi-path Distance Vector (AOMDV)}

Ad hoc On-Demand Multi-path Distance Vector is an extended version of AODV but with better performance. AOMDV finds loop-free and disjoint multiple paths. It provides link disjoint path as well as node-disjoint path with additional restrictions. However, maintaining the node-disjoint path will limit the number of suitable alternate paths that can be found.

\section{S-AOMDV}


S-AOMDV is a multipath routing protocol which is designed for VANET on the basis of AOMDV. The route discovery and route failure management is similar to AOMDV. The major difference between AOMDV and S-AOMDV is that, with extra packets, S-AOMDV can detect the average speed of all the nodes in the range and hop count in each path and based on this information S-AOMDV selects the primary transmission route. These extra packets increase the traffic in the network and cause underutilization of bandwidth.

\section{AODVM}

AODVM is again a modified version of AODV but the way this protocol constructs the routes is different from AODV. Each node records the neighbor table to find the route and updates the table on receiving the request messages. This protocol ensures that each node will join only one route while transmission.

\subsection{Summary}

This chapter introduced VANETs many challenges, the propagation, mobility models, VANET routing strategies and existing algorithms. In this thesis I will be using Nakagami radio propagation model and mobility model generated using VanetMobiSim. Since the zone based ACO routing algorithm is a multipath routing algorithm, I will compare it to AODV, AMODV and GPSR which are multipath, proactive routing algorithms. 


\section{Chapter 3}

\section{Ant Colony Optimization}

Problem solving approaches that take their inspiration from nature (the social behavior of insects and other animals) are termed as Swarm Intelligence (SI). Ant Colony Optimization is one of the significant SI technique that has been widely applied in providing solution to static and dynamic problems [BDT99].

\subsection{Ant Behavior}

The behavior of ants have been studied by Goss [GADP89] which shows that ants are able to determine, over time, the shortest path from their nest to a food source and also adapt easily to path disruptions that may occur. This behavior is explained using Figure 3.1. As ants move from source to destination they deposit a chemical substance known as pheromone on their path which evaporates overtime. As more ants come along, they are attracted by pheromone and continue along the trails of the previous ants. The ants are usually attracted towards the higher concen- 
tration of pheromones which enables them to choose shortest path since these would retain a higher concentration each time than longer paths. This process of indirect communication is an example of stigmergy.

Figure 3.1: Ants behavior
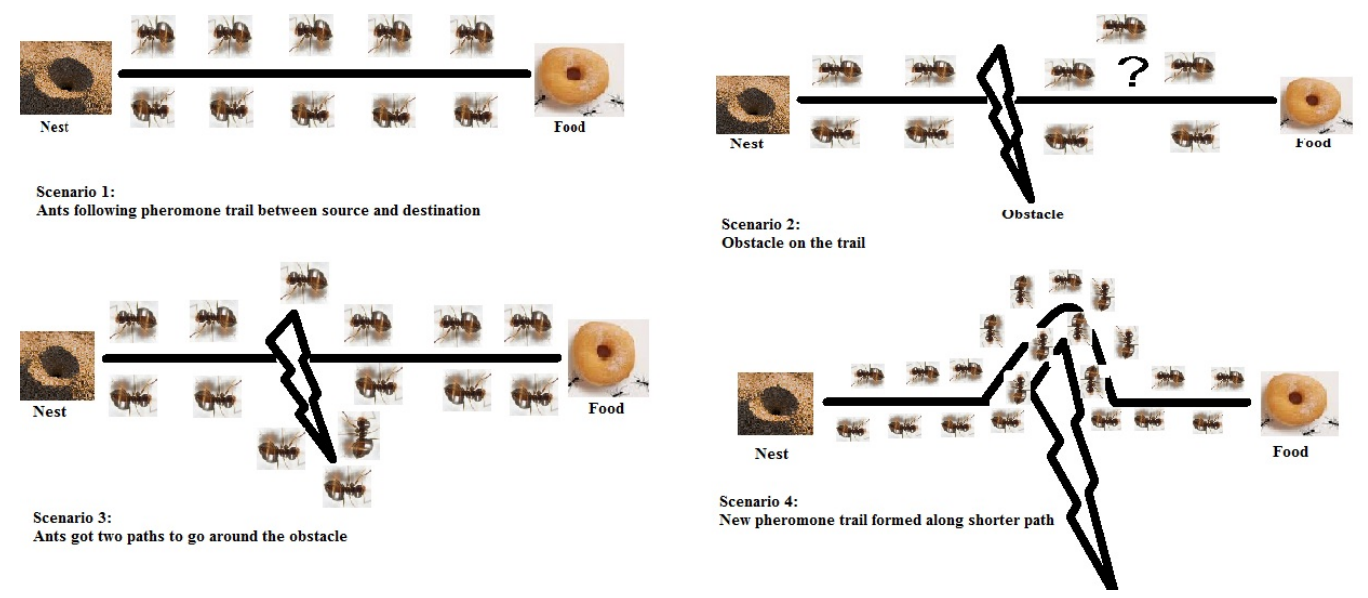

\subsection{Ant Based Algorithms}

This section describes the Ant Colony Optimization (ACO) algorithms for ad hoc network and VANETs.

\subsubsection{Ant Based Algorithms for Ad hoc network}

Ant Based Control (ABC) [SBHR96] was the first proactive, single path algorithm that was designed using ACO for wired networks. The algorithm was used to load balance and to optimize the network performance.

Antnet [CD98] is a simple extension of ABC [SBHR96] algorithm. It's a proactive algorithm for wired networks, where the route is maintained even if there is no data 
to be sent. The algorithm uses forward ants and backward ants to collect the network congestion and delay information. The forward ants are sent towards the destination at regular time intervals. Once the forward ant reaches the destination the backward ants move backwards towards the source for creating the reverse path. Forward ants are responsible for finding the minimum cost path whereas backward ants are responsible for updating the nodes' routing tables.

Based on Antnet, Gunes et al. developed Ant colony based Routing Algorithm (ARA) [GSB02], an efficient, adaptive and scalable reactive algorithm. It uses forward ants and backward ants, which are identified by unique sequence numbers. ARA is useful for reducing the routing overhead, as it works in three phases, namely: route maintenance, route discovery and route failure handling. The forward and the backward ants make the route discovery possible. Data packets are used in the route maintenance phase, where the packets maintain the route information between the source and destination. When the source receives notification about the route failure, it re-initiates the route discovery process.

The Ant Routing Algorithm for Mobile Ad hoc Network (ARAMA) [HS03] is yet another efficient and scalable proactive routing algorithm, which reduces the route discovery time, the overhead maintenance cost and delay, by reducing the forward ant generation rate. The forward ants not only keep track of the hop count, but also consider nodes' battery power and queue delay. ARAMA uses a data structure called grade which is calculated by each backward ant. Using this grade information, the backward ant updates the nodes' routing table. The algorithm uses the same grade to update pheromone value of all links. 
Co-operative Asymmetric Forward (CAF) [HSGK98] is a reactive algorithm that finds the path on demand. Here forward ants records the launch time and collects the information about the nodes in the network based on the routing tables maintained by each node. Backward ants retrace the forward ants path and deposit the pheromone on the nodes in opposite direction which helps in next hop selection.

Position Based Ant Colony Routing Algorithm (POSANT) [KO07] is a reactive algorithm, which combines the information about the position of the nodes with the ant colony optimization technique. In this algorithm, the node is always aware of its own position, the position of its neighbor and the destination position. POSANT can find an optimum route in the network, with nodes of different transmission ranges. The route is searched only when there are data packets to be sent. Each node maintains a table for storing the pheromone trail, which is used for routing purposes. The algorithm is very scalable, as it establishes the route in a short time by using a small number of control messages.

The Improved Ant Colony Optimization routing algorithm (PACONET) OTT08 is a reactive algorithm, which considers mobility, route maintenance and route failure handling. This algorithm uses forward ant for exploring the paths in the restricted broadcast manner to identify the routes from the source to the destination. Backward ants establish the path information collected by the forward ant.

AntHocNet [DCG05] is a hybrid algorithm, which combines the features of both reactive and proactive algorithms. The path setup is done reactively on demand, whereas the path probing, path maintenance and path improvement are done proactively. The source node detects a cycle by sending the forward ants and receiving 
back the backward ants. Each node maintains a routing table, which is checked when a source wants to send a packet to a destination. If the table contains the destination information, then the packets are forwarded using that route; otherwise the forward ants are broadcasted. The maintenance of the route is expensive, as the forward ants flood the network. In addition, each node stores the table with all possible destination routes, resulting in scalability issues.

Hybrid ant colony Optimization routing algorithm for mobile ad hoc Network (HOPNET) [WOTT09] is a hybrid algorithm, which combines the concept from both proactive and reactive routing algorithms. In this algorithm the network is divided into zones, and the size of the zone is measured by the radius length. Nodes are divided into interior nodes and boundary nodes. Boundary nodes are responsible for the communication between the zones. Each node maintains inter zone and intrazone routing table. The intra-zone table is maintained proactively by periodically sending the forward ants for detecting topology changes and link failures. The inter zone maintains a path to the nodes beyond its zone boundaries. The inter zone is used when the destination address is not identified within the zone. This algorithm is scalable and also handles link failures through localization.

Prasad et al. proposed a probabilistic Ant Routing (PAR) [PSR09] is a hybrid algorithm, where each node maintains the information about its neighbors, based on the HELLO message received. The forward ants are routed on the normal priority queue to explore the network and collect the network traffic information. The forward ant is unicasted if source to destination route is already present, if there is no route present between source to destination; forward ant is broadcast to identify the route. 


\subsubsection{Ant Based algorithms for VANET}

To my knowledge, Mobility-aware Ant Colony Optimization Routing DYMO (MARDYMO) CCC11 algorithm is the only nature-inspired research work done on VANET. In this, the ACO algorithm uses the nodes' position and the speed information available in the vehicular network to design a stable ant based algorithm. MAR-DYMO was designed by combining the existing Dynamic Manet On-Demand (DYMO) protocol and ACO algorithm. DYMO is a unicast reactive routing protocol and is a successor of AODV. Route discovery and Route maintenance are the two main phases of DYMO. Route discovery occurs when the source node requires sending a packet to the destination node and route maintenance helps to maintain valid routes in the routing table. When the routes life time expires, it's removed from the routing table and only routes that are active are kept. MAR-DYMO is designed by turning the DYMO into ACO by introducing the pheromone concept. In MAR-DYMO, the route request message is modified by adding additional information, such as speed, position, time stamp, displacement and scalar speed. In MAR-DYMO, route request packets are sent when required in a periodic fashion. In order to retrofit the protocol for the ACO algorithm, the routing table is modified to carry the pheromone level information and the evaporation rate associated with each route.

Table 3.1 summarizes the list of an existing ant based routing algorithms for Mobile Ad hoc Network (MANET) and VANET. 


\subsection{Summary}

In this chapter we describe the ant based routing algorithms for the MANET and VANET. While there has been few works on ACO algorithm for MANET there is very little work on ant based routing algorithms for VANET. The existing algorithm MARDYMO is a reactive algorithm for VANET whereas; the proposed MAZACORNET is a hybrid algorithm. The next chapter describes MAZACORNET. 
Table 3.1: Ant based routing algorithms with proposed year [SBHR96]-[KO07]

\begin{tabular}{|c|c|c|}
\hline Proactive Algorithms & Reactive Algorithms & Hybrid Algorithm \\
\hline $\begin{array}{l}\text { Ant Based Colony (ABC) } \\
1997\end{array}$ & $\begin{array}{l}\text { Co-operative Asymmetric } \\
\text { Forward (CAF) } 1998\end{array}$ & ANT-AODV 2002 \\
\hline Ant net 1997 & $\begin{array}{l}\text { Ant Colony Based Routing } \\
\text { Algorithm (ARA) } 2002\end{array}$ & Anthoc net 2004 \\
\hline $\begin{array}{l}\text { Routing By Ants (RBA) } \\
1998\end{array}$ & $\begin{array}{l}\text { Antbased Distributed Rout- } \\
\text { ing Algorithm (ADRA) } \\
2004\end{array}$ & $\begin{array}{l}\text { Multicast for Ad hoc Net- } \\
\text { work with Hybrid Swarm } \\
\text { Intelligence } \\
2007\end{array}$ \\
\hline $\begin{array}{l}\text { Ant Routing Algorithm for } \\
\text { Mobile Ad hoc Networks } \\
\text { (ARAMA) } 2003\end{array}$ & $\begin{array}{l}\text { Ad hoc Networking with } \\
\text { Swarm Intelligence (ANSI) } \\
2005\end{array}$ & $\begin{array}{l}\text { Hybrid Ant Colony Op- } \\
\text { timization Routing Algo- } \\
\text { rithm for Mobile Ad hoc } \\
\text { Network (HOPNET) } 2008\end{array}$ \\
\hline $\begin{array}{l}\text { Mobile Ant Based Routing } \\
\text { (MABR) } 2003\end{array}$ & $\begin{array}{l}\text { Ant Colony Based Multi- } \\
\text { path QoS aware Routing } \\
\text { (AMQR) } 2005\end{array}$ & $\begin{array}{l}\text { Ant Colony Optimization- } \\
\text { Ants Hybrid Routing } \\
\text { (ACO-AHR) } 2008\end{array}$ \\
\hline TERMITE 2003 & $\begin{array}{l}\text { Multicast for Ad hoc Net- } \\
\text { work with Swarm Intelli- } \\
\text { gence (MANSI) } 2005\end{array}$ & $\begin{array}{l}\text { Probabilistic Ant Routing } \\
\text { (PAR) } 2009\end{array}$ \\
\hline \multirow[t]{7}{*}{$\begin{array}{l}\text { GPS/Ant-Like (GPSAL) } \\
2006\end{array}$} & $\begin{array}{l}\text { Position based Ant colony } \\
\text { routing (POSANT) } 2008\end{array}$ & $\begin{array}{l}\text { Fuzzy Ant Colony Based } \\
\text { Routing (FACO) } 2009\end{array}$ \\
\hline & $\begin{array}{l}\text { Improved Ant Colony Op- } \\
\text { timization algorithm (PA- } \\
\text { CONET) } 2008\end{array}$ & Accordant 2009 \\
\hline & $\begin{array}{l}\text { PSO based On Demand } \\
\text { Multicast Routing Protocol } \\
\text { (PSO-ODMRP) } 2008\end{array}$ & MAARA 2010 \\
\hline & $\begin{array}{l}\text { Ant-based Energy-aware } \\
\text { Disjoint Multipath Routing } \\
\text { Algorithm } \\
2011\end{array}$ & $\begin{array}{l}\text { Ant Tabu Based Routing } \\
\text { Algorithm (ATBRA) } 2010\end{array}$ \\
\hline & $\begin{array}{l}\text { A Novel on Demand Ant } \\
\text { Based Security Alert Rout- } \\
\text { ing Algorithm (ODASARA) } \\
2011\end{array}$ & $\begin{array}{l}\text { Efficient Ant } \quad \text { Routing } \\
\text { (ANT-E) } 2010\end{array}$ \\
\hline & $\begin{array}{l}\text { A New On-Demand Ant- } \\
\text { Based Multiagent routing } \\
\text { Algorithm (SAMP-DSR) } \\
2011\end{array}$ & $\begin{array}{l}\text { Optimized Ant Based Rout- } \\
\text { ing (ODAMARA) } 2011\end{array}$ \\
\hline & & AntNet-RSLR 2011 \\
\hline
\end{tabular}




\section{Chapter 4}

\section{An Improved Mobility Aware Zone}

\section{based Ant Colony Optimization}

\section{Routing for VANET}

This chapter describes a hybrid ACO based routing algorithm for VANET.

\subsection{Link Stability}

Location management and connection management are important issues to consider while designing routing algorithms. Commonly, location management is done by means of using the Global Positioning System (GPS), which provides vehicles position and speed. I have used GPS in my work. Connection management, which maintains stable routes between the vehicles, needs to be calculated. This translates to determining the link stability, an important parameter to make intelligent data 
Chapter 4: An Improved Mobility Aware Zone based Ant Colony Optimization Routing for VANET

forwarding decisions for overall routing performance.

Let us assume $i$ and $j$ are two vehicles on the road. By using GPS, I obtain the initial positions of $i$ and $j$ denoted as $\left(X_{i 0}, Y_{i 0}\right)$ and $\left(X_{j 0}, Y_{j 0}\right)$ as well as their initial speed, $V_{i 0}$ and $V_{j 0}$, respectively. If the vehicles are in the same communication range, they will be assumed to be neighbors. I have used $D$ to denote the distance between the two vehicles and $R$ to denote the communication range. If $D \geq R$, the link between the vehicles will break. The lifetime $(\Delta t)$ of the link ij from current time, $t_{1}$, to initial time, $t_{0}$, when $\mathrm{D}=\mathrm{R}$ is $\Delta t$. Given, the initial position of vehicles and speed information, The lifetime $(\Delta t)$ is calculated using the distance formula given below Gow82].

$$
D^{2}=\left\|\left(\mathrm{X}_{\mathrm{i} 0}+\mathrm{V}_{\mathrm{i} 0} \Delta \mathrm{t}\right)-\left(X_{j 0}+V_{j 0} \Delta t\right)\right\|^{2}+\left\|\left(\mathrm{Y}_{\mathrm{i} 0}+\mathrm{V}_{\mathrm{i} 0} \Delta \mathrm{t}\right)-\left(Y_{j 0}+V_{j 0} \Delta t\right)\right\|^{2}
$$

The Link Stability (LS) of link $i j$ [MLF07] is calculated using the following Equation:

$$
L S=\frac{\Delta t_{i j}}{t_{\max }}
$$

Here, $t_{\max }$ is a constant parameter and corresponds to the validity period of the time of the routing table. In my algorithm, this link stability parameter will be used to make data forwarding decision resulting for overall better routing performance. 


\subsection{Ant colony-based Algorithm for VANET}

The pheromone parameter is the most important component of the ACO algorithm. Recall that a pheromone is the scent deposited on the ground to attract more ants to follow their path. For VANET, the increment and decrement (evaporation) of the pheromone concentration on the link provides information on the quality of the link, in particular lifetime of the link. To identify the quality of the link, I find the amount of pheromone deposited by an ant along its path from source to destination on link $i j$. The calculation of pheromone deposit, $\Delta \phi_{i j}$, on link $i j$ depends on the Link Stability (LS) of $i j$ and the probability of successfully receiving the message $P_{R}$ as provided in Equation 4.3

$$
\Delta \phi_{i j}=P_{R}+L S
$$

where LS is the link stability calculated in 4.2 and $P_{R}$ is the probability of successfully receiving the message.

The probability of successfully receiving the message depends on the distance between the vehicles lying within same communication range and can be estimated by using the Nakagami Fading Model [Mor09]. This model captures the dynamics of VANET in highway [TJM ${ }^{+}$04] and urban [RRC07] scenarios and the probability is given by

$$
P_{R}(D, R)=e^{-m\left(\frac{D}{R}\right)^{2}} \sum_{i=1}^{m} \frac{\left(m\left(\frac{D}{R}\right)^{2}\right)^{i-1}}{(i-1) !}
$$

where $m$ is the fading parameter. For example if $m=3$ it implies a fast-fading parameter or moderate radio conditions.

Using Equations (4.3) and 4.4, the pheromone deposit on link $i j$ is calculated 
as:

$$
\Delta \phi_{i j}=e^{-m\left(\frac{D}{R}\right)^{2}} \sum_{i=1}^{m} \frac{\left(m\left(\frac{D}{R}\right)^{2}\right)^{i-1}}{(i-1) !}+L S
$$

Pheromone Evaporation: In the ACO algorithm, the evaporation rate, $\phi$ GSB02] is usually set as a constant parameter. However, in my algorithm, I have considered different evaporation rates for each and every link based on the estimated link lifetime. Therefore, the evaporation on each link $i j$ is represented as $\rho_{i j}$ [CCC11]:

$$
\rho_{i j}=1-\left(\frac{\epsilon}{\phi}\right)^{\frac{t_{e v}}{\Delta t}}
$$

where $\epsilon$ is the least amount of pheromone associated with link and $\frac{t_{e v}}{\Delta t}$ is the reciprocal of the number of times evaporation is performed.

Now that the ACO algorithm for routing in VANET has been explained, I will describe my hybrid Mobility Aware Zone based Ant Colony Optimization Routing for VANET. This algorithm is scalable and uses the benefits of reactive and proactive routing algorithms.

\subsection{Zone-based ant colony VANET algorithm}

In my algorithm the network is divided into zones. For routing the packets, I follow a proactive approach within the zone and a reactive approach between the zones. The radius length measured in hops determines the size of the zone. A vehicle can exist within two overlapping zones and the zones can vary in size. A vehicle is categorized as interior vehicle, boundary vehicle and exterior vehicle. All the vehicles within a zone having a hop distance of less than the radius are known as interior 
vehicles. The overlapping vehicles within the zones with the hop distance equal to the radius are known as boundary vehicles and the vehicles with the hop distance greater than the radius are known as exterior vehicles.

Figure 4.1 explains about the vehicles with an example. If the source vehicle is $\mathrm{S}$ and the radius of the zone is 2 then vehicles A, D, F are boundary or border vehicles, and vehicles B, C, E are interior vehicles. All other vehicles are exterior vehicles i.e. the vehicles outside the zone.

Figure 4.1: MAZACORNET example

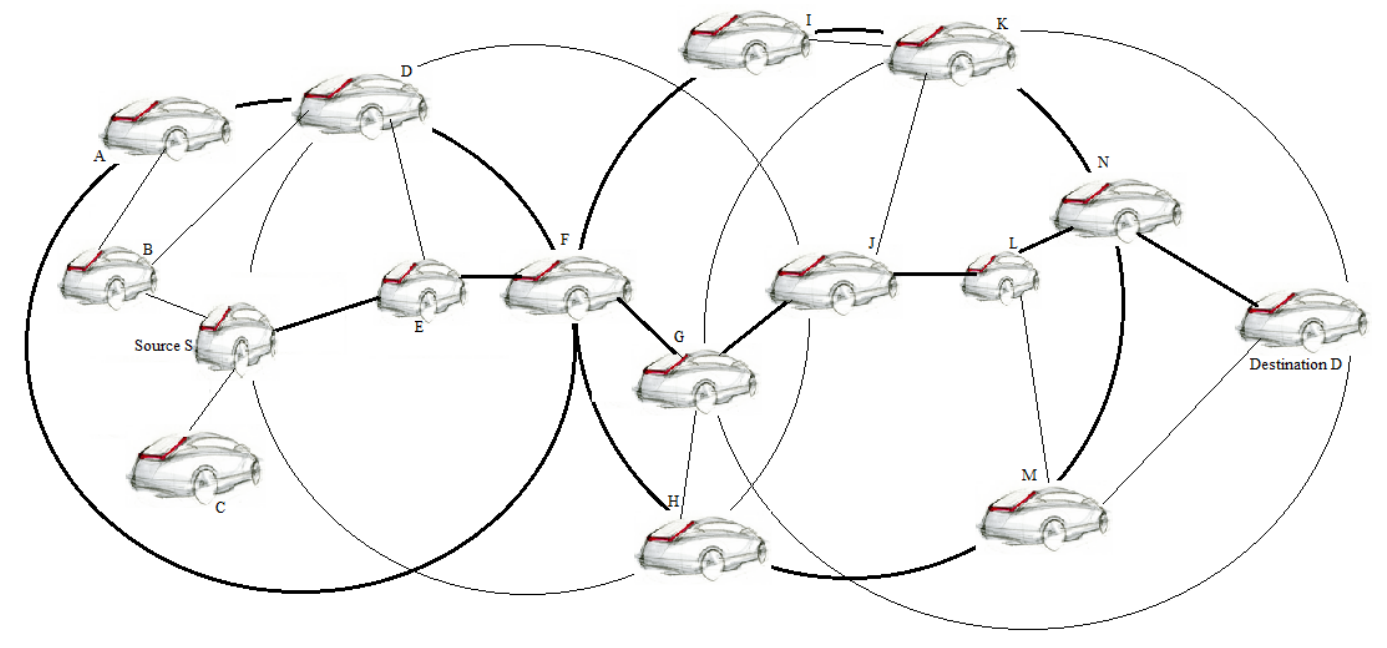

The two main phases of routing, route discovery and route maintenance can either happen within the zone or between zones. I have used two routing tables: Intra zone routing table and Inter zone routing table. The Intra zone routing table proactively updates the information within the zone, whereas, the Inter zone routing table tracks the information between the zones, on demand. During route discovery and maintenance, I have used five different types of ants. These are: internal forward ants, external forward ants, backward ants, notification ants, error ants. The data struc- 
ture of the ant contains Source, Destination, Sequence number, Type, Hops, Speed, Position and Path.

- Source: Source node address is stored in source field.

- Destination: This field stores the destination address. This field is left blank for internal forward ant and stores the destination nodes address for external forward ants.

- Sequence Number: Each ant is tagged with a sequence number, stored under sequence number field.

- Type: There are five types of ants which are described in the Type field. 0 is internal forward ant, 1 is external forward ant, 2 is backward ant, 3 is notification ant, 4 is error ant.

- Hops: It is used to indicate the number of hops between the node and all the nodes within its zone. This field helps to differentiate between a peripheral node and an internal node. For internal forward ant, the zone radius is assigned and for external forward ant, I leave this field blank.

- Speed: It is the speed of the vehicle.

- Position: This field contains the current position of the vehicle.

- Path: Path field represents the sequence of nodes between source and destination.

The phases are explained below as: 


\section{Route discovery within the zone}

The Intra zone routing table is used for route discovery within the zone. This table will contain the information about all the vehicles within the zone and the internal forward ant periodically updates the vehicle's information in the table. In this routing table, I have incremented and decremented (evaporate) the pheromone concentration on the vehicles based on the identified route, using Equations 4.5 and 4.6.

\section{Route discovery between the zones}

When the vehicle fails to find the destination with the zone, Inter zone routing table is used to identify the new route using the boundary vehicles. External forward ants are sent to the boundary vehicle for route discovery which will in turn forward to the next boundary vehicle from its current location, until the destination vehicle is found. If the destination vehicle is found, then the backward ant will traverse back to the source, based on the inherited route. However, if the destination is not within the current vehicle's zone or the path's lifetime has expired, the route discovery process will be repeated using the boundary vehicle from the current zone.

\section{Route maintenance}

Route paths may break because of the dynamic nature of the network. If the broken route is within the internal zone, then it is repaired periodically using the proactive approach, but if the Inter zone route breaks, then the upstream vehicle of the broken link is used to store the packets and find an alternative path. When the 
alternative route is identified, then a notification ant is used to update the new route on the source vehicle. If an alternative route is not identified, then an error ant is used to notify the source vehicle about route failure.

\subsection{Summary}

This chapter described my Mobility Aware Zone based Ant Colony Optimization Routing for VANET, called MAZACORNET. I test my algorithm through simulations in the next chapter. 


\section{Chapter 5}

\section{Simulation Results}

In this chapter to evaluate the performance of my algorithm, I analyze the results obtained from large number of simulations run on NS2.

\subsection{Simulation environment}

I implemented MAZACORNET using NS2 simulator [L11]. VanetMobiSim, NS2 and AWK are the three open source tools which are used during the simulations. These tools are described in more detail below.

\section{VanetMobiSim}

The Vehicular Ad Hoc Network Mobility Simulator (VanetMobiSim) [FHFB07, is an extension to CanuMobiSim [Ste05]. This extensible framework includes number of mobility models and also parsers for geographic data sources in different formats. Currently, VanetMobiSim provides two mobility models Vehicular spatial model and Vehicular-oriented mobility model. Vehicular spatial model is composed of spatial 
elements such as traffic lights or multi-lane roads whereas vehicular-oriented mobility model manages intersections and vehicles acceleration and decelerations. VanetMobiSim provides great flexibility in creating more realistic traffic scenarios. For generating a real traffic scenario using the XML files, I have installed VanetMobiSim, CanuMobiSim, Java development Kit and Apache Ant v1.7.

\section{NS2}

The network simulator, NS2, is a network simulator which provides packet level simulation over several transport protocols, wired networking, ad hoc routing protocols, satellite and so on. NS2 can simulate mobile nodes and the mobility of the nodes is specified either in simulation file or in mobility trace file. In my simulation this trace file is generated using VanetMobiSim. NS2 was developed in $\mathrm{C}++$ and provides simulation interface through Object Oriented dialect of Tool command language $(\mathrm{OTcl})$. In my research I have described the network topology by writing OTcl script. Since the simulation kernel code is available I could implement my routing algorithm on NS2. I installed NS2.34 after downloading and extracting it in personal directory.

\section{Awk}

Awk is a data extraction and reporting tool which I used for the purpose of producing formatted reports.

\subsection{Simulation Process}

Figure 5.1 defines the VANET simulation process. First I defined the mobility scenario in a XML file, then launched VanetMobiSim framework to produce a vehicle 
mobility trace file in NS2 format containing time, velocity and positions of the vehicles. Then I incorporated this file to communication definition file, implemented in Tcl language and ran the simulation. This gave me NS2 trace file with all the routing events that occurred during simulation. Finally I used the AWK tool to filter the event trace file to extract all the significant data required for the evaluation of my algorithm.

Figure 5.1: VANET Simulation Process

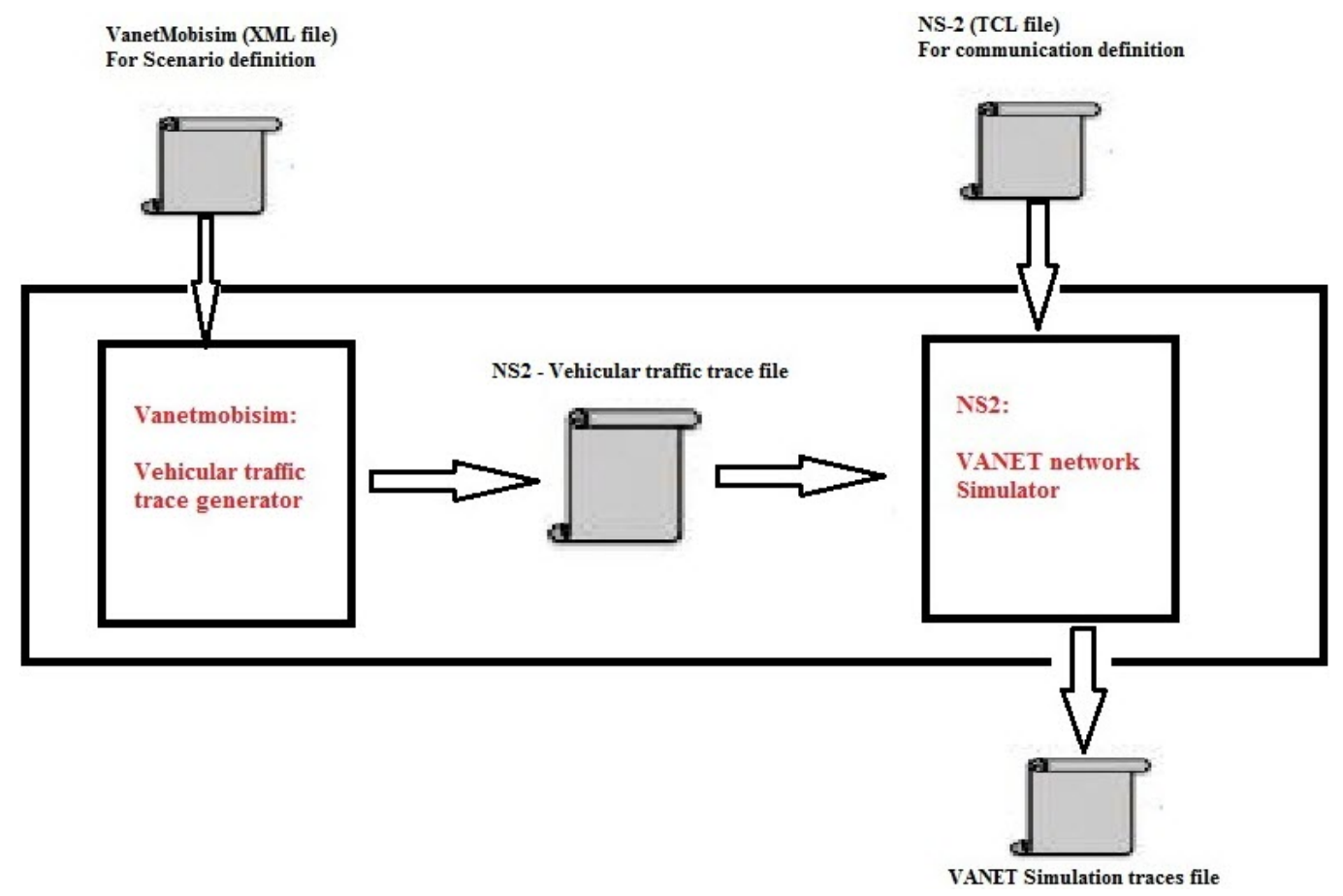

\subsection{Simulation Setup}

During simulation I used urban traffic scenario with random street configuration. Initially I started with 25 vehicles in the $500 \times 500 \mathrm{~m}^{2}$ area, including 10 traffic lights with step of 20 seconds and later gradually increased the vehicles to 100 in the 1500 
$\mathrm{x} 1500 \mathrm{~m}^{2}$ area. Following is the network configuration of the simulations in NS2:

- Simulation time is set to 2000 seconds. Vehicles motion starts at $t=0$ s and transmission starts at $\mathrm{t}=100 \mathrm{~s}$.

- Number of vehicles has been set to 25, 50, 75 and 100.

- In all simulations there are only one source node and one destination node.

- Packet Length is 512 bytes.

- The queue selected is PriQueue which gives priority to routing packets. The queue size is set to 50 packets.

- Omni-directional antennas are selected with height of $1,5 \mathrm{~m}$.

- IEEE 802.11b and IEEE 802.11p was selected as MAC protocols.

- Nagakami propagation model was selected

- MAZACORNET is the routing protocol used in the simulations.

- Other algorithms used for comparison are AODV, AMODV, GPSR.

\subsection{Simulation Metrics}

To analyze the efficiency and effectiveness of my routing algorithm I used the following six metrics:

- Average end-to-end delay: This metric shows the time required for a packet to go through the network from source node to destination node. It characterizes the latency generated by the routing approach. 
- Packet delivery ratio: The metric shows the ability of routing protocol to transfer end-to-end data successfully. It is the percentage of data packets received by the destination relative to data packets sent by the source node.

- Data throughput: This metric considers the ratio between the total bits that source node is able to inject to the network and reach destination node within a particular time frame. For example, if $x$ number of bits are delivered within $t$ time at a node then the throughput at the node can be defined as $\frac{x}{t}$.

- Routing Overhead: This metric measure the additional traffic generated by the routing protocol for packets successfully delivered. It is defined as the number of extra duplicate routing packets per number of data packets received at destinations.

- Scalability: This metric measures the capability of an algorithm in handling a growing number of nodes.

\subsection{Simulation Results}

In this section, I have compared the performance of MAZACORNET with other existing routing algorithms. Figure 5.2 shows the end to end delay of MAZACORNET in comparison to AODV, AMODV and GPSR. MAZACORNET produces better results than other algorithms. This is because of the zone framework and the local intrazone routing table and interzone routing table. In intrazone table routes are maintained proactively within the zone whereas, in interzone the path's recently visited by ants are stored. Since, paths are readily available this helps in achieving fast 
end to end packet transmission. In MAZACORNET, we can adjust the evaporation rate based on Equation 4.6. So, by adjusting the evaporation rate of pheromone on the link broken links can be easily discarded by ants. These reasons allow MAZACORNET to produce better end to end delay results.

Figure 5.2: End to End Delay: MAZACORNET vs Other routing algorithms

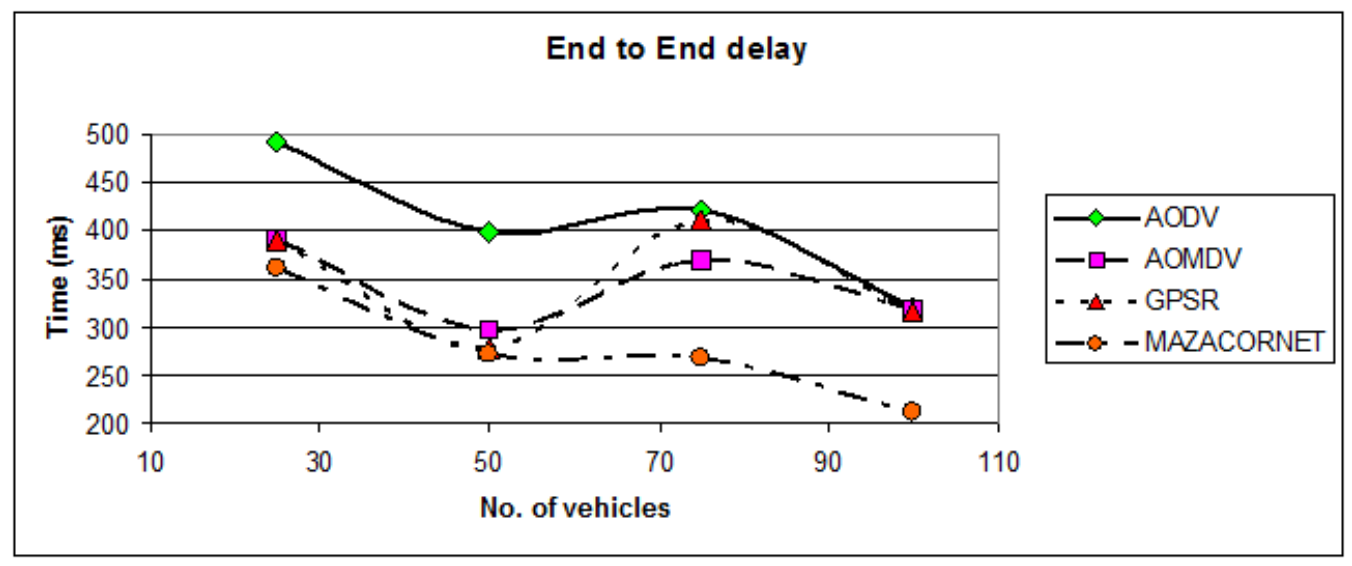

Figure 5.3 shows the delivery ratio for MAZACORNET and other existing routing algorithms (AODV, AMODV, GPSR). When there are fewer vehicles in the network MAZACORNET did not show a good delivery ratio. This can be attributed to the intermediate vehicle or boundary vehicle that could not deliver packets because of less pheromone concentration on the link as a result of moving vehicle. In this scenario the upstream vehicle buffers all the packets and use repair procedure to find the alternative path to the destination. Once new path is discovered a notification ant is sent towards source vehicle new route. Also, buffered packets are delivered to destination node using this newly identified route. In other scenario if all upstream and downstream vehicles are moved away then data packet is dropped. Note that as the network size increases and with more neighbors for a vehicle, the delivery ratio 
for MAZACORNET is better than other algorithms. This is because the ants can choose from multiple paths rather than a single path as in AODV and GPSR.

Figure 5.3: Packet Delivery Ratio: MAZACORNET vs Other routing algorithms

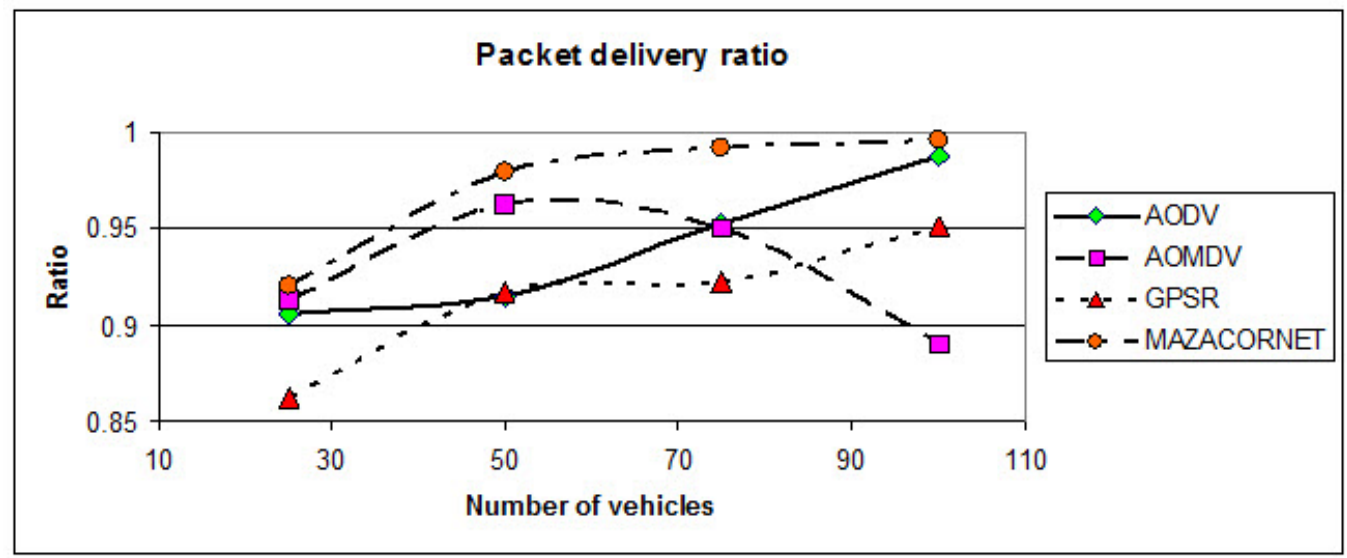

Figure 5.4 shows the routing overhead of MAZACORNET and other routing algorithms. AODV, AOMDV are pure reactive protocol with no concept of zone. Whereas, MAZACORNET is proactive within a zone. The routes within the zone are maintained by periodically sending the control packets. This is a major reason of the overhead in MAZACORNET. When the network size increases a vehicle has more choices for paths to destination which proves the algorithm to be multi path algorithm. 
Figure 5.4: Routing Overhead: MAZACORNET vs Other routing algorithms

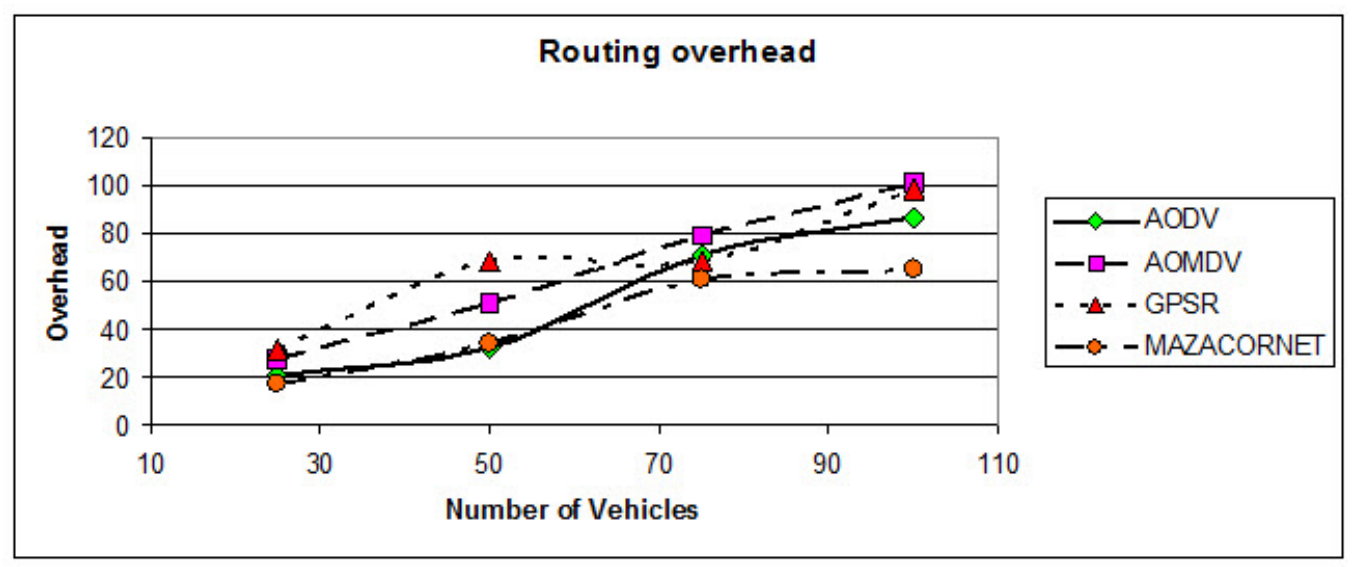

The next evaluated metric is data throughput. Figure 5.5 shows the evolution of data throughput throughout the simulation. Here, the number of vehicles is varied keeping simulation area and radius of the zones constant. Therefore, increase in the number of vehicles increases the number of vehicles within a zone creating a higher concentration of the vehicles within a zone. So when ant traverses in a zone with higher concentration, the probability of getting lost decreases which increases throughput. These results are highly related to those discussed for the packet delivery in Figure 5.3, as higher the received packet ratio, the higher is the data throughput. 
Figure 5.5: Throughput: MAZACORNET vs Other routing algorithms

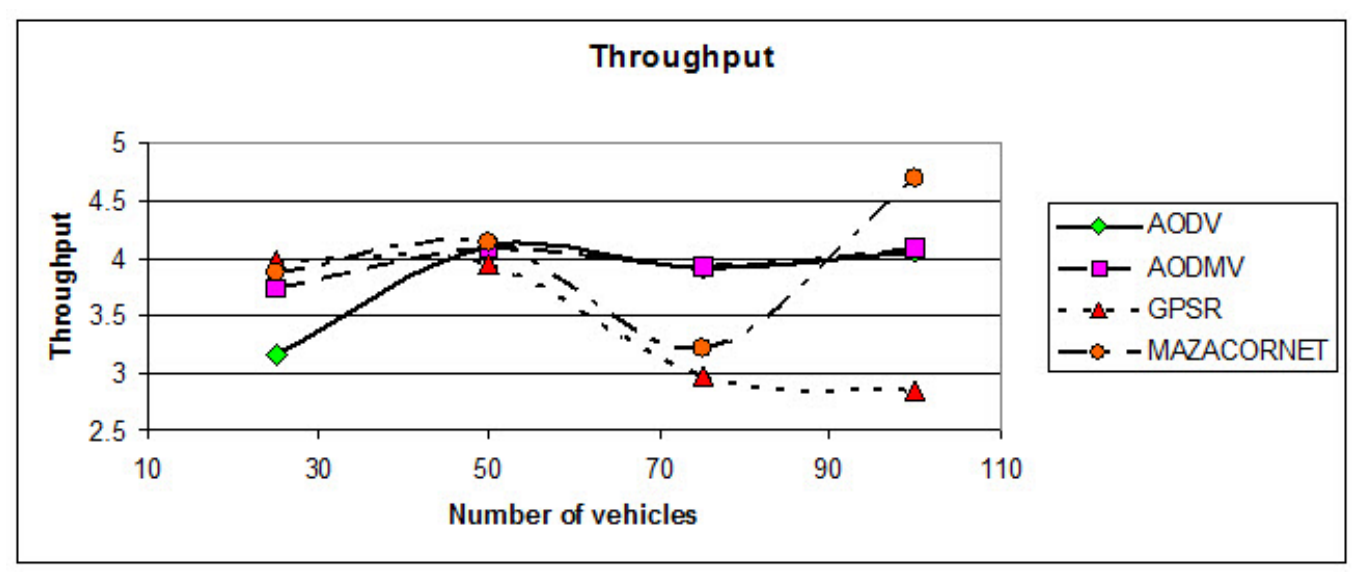

Next we observe the packet delivery ratio, routing overhead and end to end delay with varying zone radius and vehicles.

In Figure 5.6 we increase the zone radius from 2 to 10 with varying vehicle count. When the zone radius is 10 and number of vehicles is 100, a vehicle is reachable from other vehicles easily. On the other hand with radius of 2 and 100 vehicles, the number of vehicles in each zone is sparse. MAZACORNET as seen before works well for dense networks as ants are able to find multiple paths. Therefore, from Figure 5.6 we can see that 100 vehicles with zone radius of 10 shows high delivery ratio. 
Figure 5.6: Packet delivery ratio with various zone radius

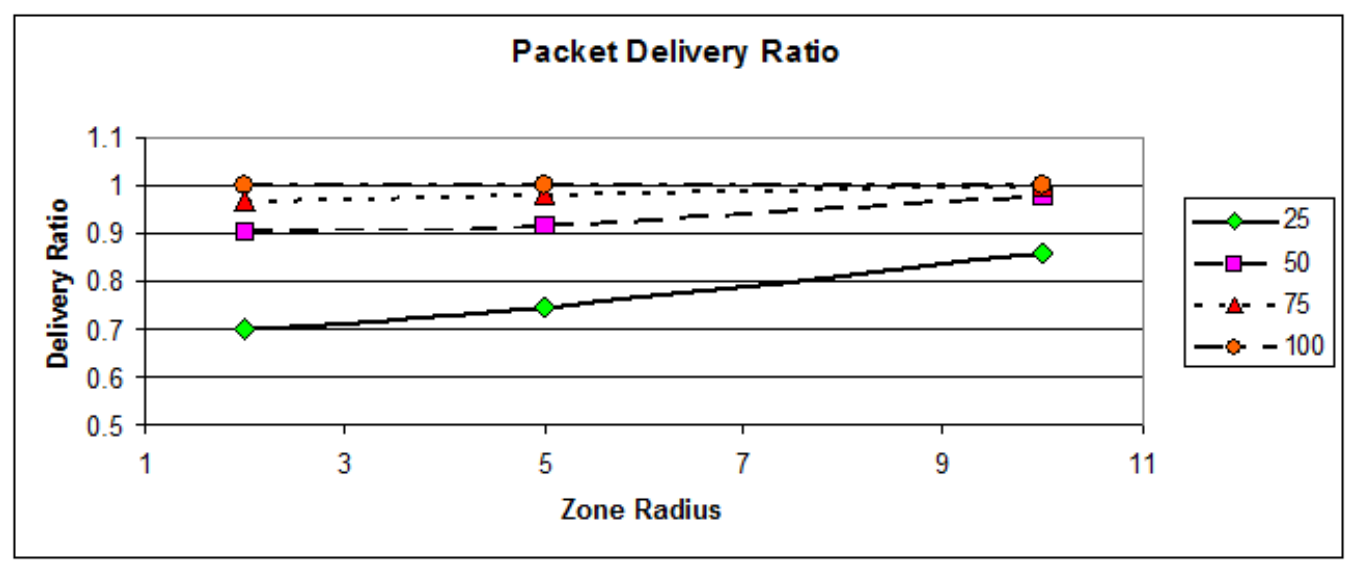

Figure 5.7 shows end to end delay for varying vehicle counts and zone radius. When the number of vehicles is 100 and zone radius is 2 , it takes more time to transmit packets across the network. Whereas in case of 100 vehicles and zone radius of 10, it takes less time to transmit a packet. This is because MAZACORNET starts to work proactively accepting a lot of vehicles within a zone. Hence, Figure 5.7 shows for large number of vehicles and larger zone radius we have better end to end delay than less number of vehicles and smaller zone radius. 
Figure 5.7: End to end delay with various zone radius

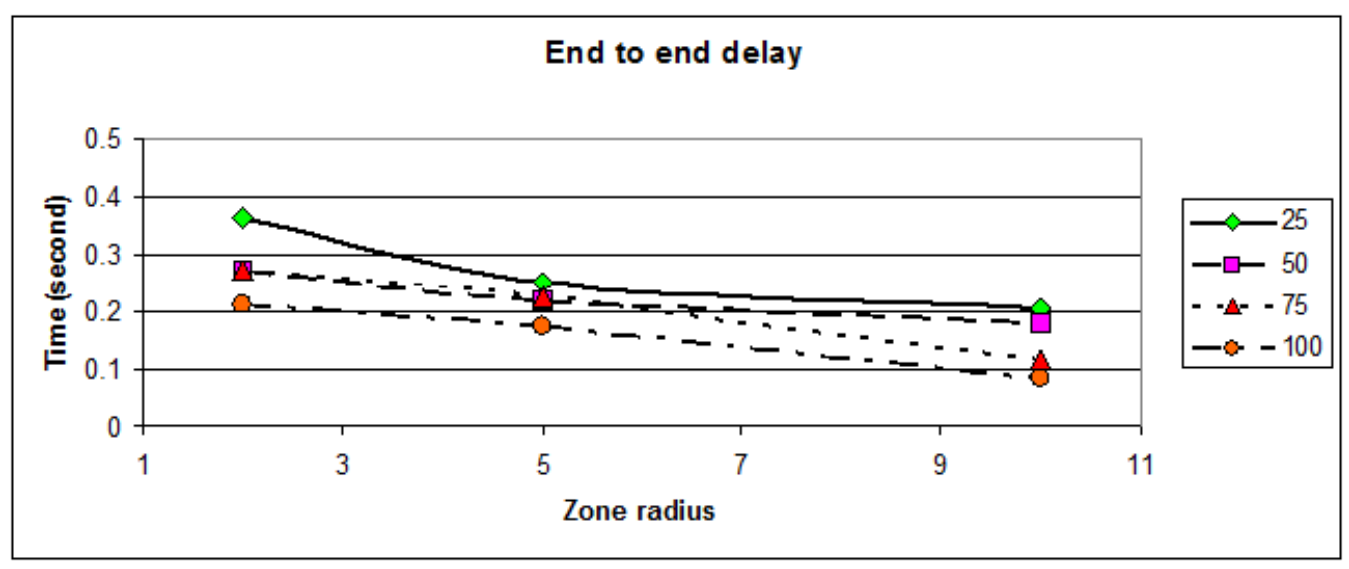

In Figure 5.8 we increase zone radius from 2 to 10 with varying vehicle count. When the number of vehicles is 100 and zone radius is 2 we have less congestion on the network. On other hand with zone radius of 10 and vehicles count of 100, we have more congestion due to more vehicles and proactive ants which keeps updating the routing table frequently. Though MAZACORNET provides good network connectivity, meaning there will be more routes available, it generates much more control traffic. Therefore, from Figure 5.8 we can see as the number of vehicles and zone radius increases we have high routing overhead. 
Figure 5.8: Routing overhead with various zone radius

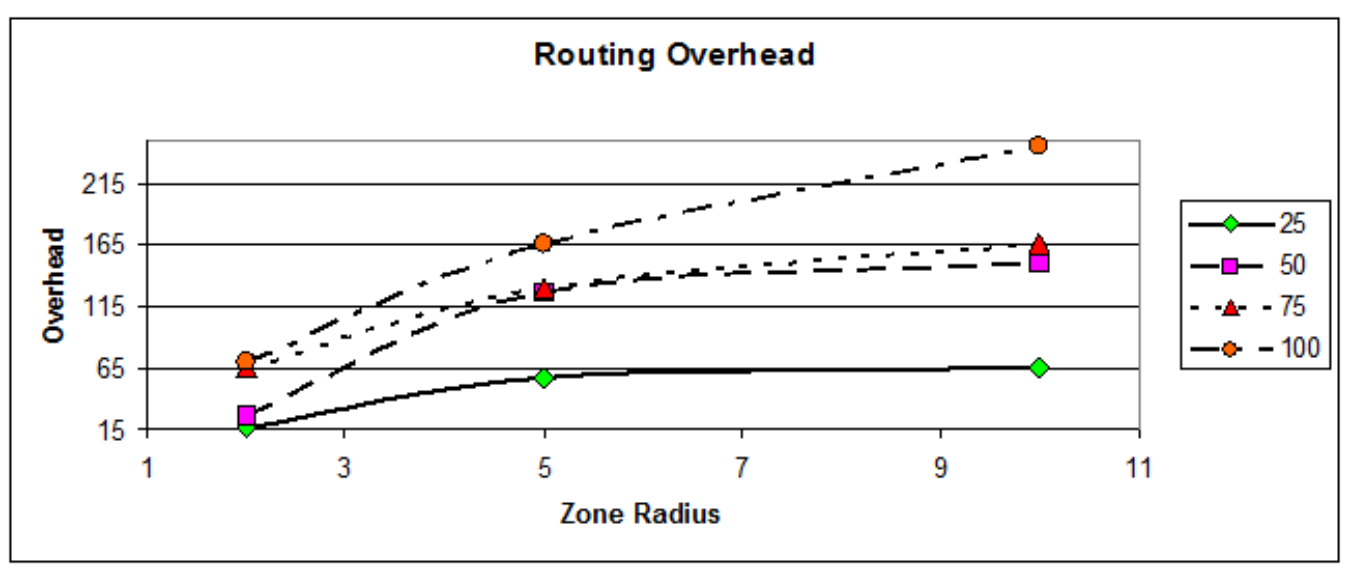

\subsection{Summary}

This chapter described the simulation process I used to evaluate my algorithm, several metrics I used to compare my algorithm with other existing algorithms and the performance of my Mobility Aware Zone based Ant Colony Optimization Routing for VANET. Results show that the algorithm is suitable for urban scenarios or in other words for dense network. When the network is dense the algorithm achieved higher delivery ratio and lesser end to end delay though routing overhead increased because of congestion. However, MAZACORNET still performed better than other existing algorithms because of good network connectivity achieved because of its multipath properties. 


\section{Chapter 6}

\section{Conclusion}

We proposed a swarm based hybrid routing algorithm for VANET. This is a multipath routing algorithm with no concept of message broadcasting. Reactive and proactive algorithm works together to find a route to the destination. One of the important issues in the ACO algorithm is modeling the dynamic environment in VANET. I use the Nagakami probability distribution function to study the dynamic data packet reception probabilities in VANET. The algorithm, subdivides the vehicles into zones. The algorithm applies proactive routing within a zone and reactive routing between zones.

The results of the algorithm show that that algorithm works well for dense networks. The algorithm produces better delivery ratio and is scalable for zones beyond four. When compared to other existing VANET algorithms, the hybrid algorithm proved to be more efficient in terms of packet delivery ratio and end to end delay. The algorithm is suitable for urban scenarios or in other words for dense network where there are more vehicles within the zone. Route maintenance strategy is used 
due to the continuous movement of the vehicles. I have used the vehicle position and speed to make predictions on the mobility of the vehicles. My thesis work focuses and emphasize on the use of ACO in the routing algorithms for VANET.

In conclusion, the hybrid ACO algorithm is scalable and achieves good network connectivity. To our knowledge is the first ant based routing algorithm for VANET that uses the concept of zones. 


\section{Chapter 7}

\section{Future Work}

Routing in VANET is more challenging than the routing in other types of adhoc network due to continuous movement of the vehicles. Work is still being done in this area to find the better routing scheme for VANET.

From the results obtained after the simulation and evaluation of my algorithm, I have learned the strength and the weakness of the algorithm. As a part of future work following can be tried to improve the algorithms performance:

Implement a network that combines not only car-to-car communication but also car-to-infrastructure communication i.e. road side units to make algorithm more suitable for the highway scenarios. Like other ant based algorithms, incorporate the concept of multicasting in the network to improve the route's search time. Different mobility models can be used which may help in considering the behavior of the driver. This work can be further extended by doing a comparison to other ant algorithms designed for quality of service, power management and other issues relating to VANETs. 


\section{Bibliography}

[AM08] Mario A Amaya and Christopher L Magee. The progress in wireless data transport and its role in the evolving internet. Nov. 2008.

[BB03] Abdelmalik Bachir and Ahderrahim Benslimane. A multicast protocol in ad hoc networks inter-vehicle geocast. In Proceedings of 5\%th IEEE Semiannual Conference on Vehicular Technology, volume 4, pages 24562460, Jeju, South Korea, April 2003.

[BCG01] R. Bruno, M. Conti, and E. Gregori. WLAN technologies for mobile ad hoc networks. In Proceedings of the 34th Hawaii International Conference on System Sciences, Maui, Hawaii, Jan. 2001.

[BDT99] Eric Bonabeau, Marco Dorigo, and Guy Theraulaz. Swarm Intelligence: From Natural to Artificial Systems. Oxford University Press, USA, Sep. 1999.

[BEH04] Jeremy J. Blum, Azim Eskandarian, and Lance J. HoffmanBlum. Challenges of intervehicle ad hoc networks. IEEE Transactions on Intelligent Transportation Systems, 5(4):347-351, Dec. 2004. 
[Bra00] Brad Karp and H. T. Kung. GPSR: Greedy Perimeter Stateless Routing for wireless networks. In Proceedings of the 6th Annual ACM International Conference on Mobile Computing and Networking, pages 243-254, Boston, Massachusetts, United States, Aug. 2000.

[BSH00] Linda Briesemeister, Lorenz Schafers, and Gunter Hommel. Disseminating messages among highly mobile hosts based on inter-vehicle communication. In Proceedings of the IEEE Intelligent Vehicles Symposium, pages 522-527, Dearborn, MI, USA, Oct. 2000.

[CB05] David R. Choffnes and Fabián E. Bustamante. An integrated mobility and traffic model for vehicular wireless networks. In Proceedings of the 2nd ACM International Workshop on Vehicular ad hoc networks, pages 69-78, Cologne, Germany, Sep. 2005.

[CBD02] Tracy Camp, Jeff Boleng, and Vanessa Davies. A survey of mobility models for ad hoc network research. Wireless Communications and Mobile Computing, 2(1):483-502, Feb. 2002.

[CCC11] Sergio Luis O. B. Correia, Joaquim Celestino, and Omar Cherkaoui. Mobility-aware ant colony optimization routing for vehicular ad hoc networks. In Proceedings of IEEE Conference on Wireless Communications and Networking Conference, pages 1125-1130, Cancun, Mexico, March 2011.

[CD98] Gianni Di Caro and Marco Dorigo. AntNet: distributed stigmergetic 
control for communications networks. Journal of Artificial Intelligence Research, 9(1):317-365, Aug. 1998.

[CGMV05] Giuseppe Caizzone, Paolo Giacomazzi, Luigi Musumeci, and Giacomo Verticale. A power control algorithm with high channel availability for vehicular ad hoc networks. In Proceedings of IEEE International Conference on Communications, volume 5, pages 3171-3176, Seoul, Korea, May 2005.

[CJ03] Thomas Heide Clausen and Philippe Jacquet. Optimized link state routing protocol (OLSR). Technical report, INRIA, Oct. 2003.

$\left[\mathrm{CWT}^{+} 08\right] \quad$ Pei-Chun Cheng, Jui-Ting Weng, Lung-Chih Tung, Kevin C. Lee, Mario Gerla, and Jerome Haerri. GeoDTN+NAV: A hybrid geographic and dtn routing with navigation assistance in urban vehicular networks. In Proceedings of International Symposium on Vehicular Computing Systems, Dublin, Ireland, July 2008.

[CXJJ09] Yufeng Chen, Zhengtao Xiang, Wei Jian, and Weirong Jiang. An improved AOMDV routing protocol for V2V communication. In Proceedings of the IEEE Intelligent Vehicles Symposium, pages 1115-1120, Xi'an, China, June 2009.

[DCG05] Frederick Ducatelle, Gianni Di Caro, and Luca Maria Gambardella. Ant agents for hybrid multipath routing in mobile ad hoc networks. In Proceedings of the 2nd Annual Conference on Wireless On-demand Network Systems and Services, pages 44-53, St. Moritz, Switzerland, June 2005. 
[DDB05] Mimoza Durresi, Arjan Durresi, and Leonard Barolli. Emergency broadcast protocol for inter-vehicle communications. In Proceedings of 11th International Conference on Parallel and Distributed Systems, volume 2, pages 402-406, Fuduoka, Japan, July 2005.

[FHFB07] Marco Fiore, Jerome Harri, Fethi Filali, and Christian Bonnet. Vehicular mobility simulation for VANETs. In Proceedings of 40th Annual Conference on Simulation Symposium, pages 301-309, Norfolk, Virginia, USA, March 2007.

[GADP89] S. Goss, S. Aron, J.L Deneubourg, and J. Pasteels. Self-organized shortcuts in the argentine ant. Naturwissenschaften, 76(12):579-581, Dec. 1989.

[Gow82] J.C. Gower. Euclidean distance geometry. The Mathematical Scientist, 7(1):1-14, May 1982.

[GSB02] Mesut Gnes, Udo Sorges, and Imed Bouazizi. ARA-the ant-colony based routing algorithm for MANETs. In International Workshop on Ad Hoc Networking in conjunction with International Conference on Parallel Processing, pages 79-85, Vancouver, B.C., Aug. 2002.

[GSPM08] Víctor González, Alberto Los Santos, Carolina Pinart, and Francisco Milagro. Experimental demonstration of the viability of IEEE 802.11b based inter-vehicle communications. In Proceedings of the 4th International Conference on Testbeds and research infrastructures for the de- 
velopment of networks $\&$ communities, pages 1-7, Innsbruck, Austria, March 2008.

[HS03] O. Hussein and T. Saadawi. Ant routing algorithm for mobile ad-hoc networks (ARAMA). In Proceedings of 22nd IEEE International Conference on Performance, Computing, and Communications Conference, pages 281-290, Phoenix, Arizona, USA, April 2003.

[HSGK98] Martin Heusse, Dominique Snyers, Sylvain Gurin, and Pascale Kuntz. Adaptive agent-driven routing and load balancing in communication networks. Advances in Complex Systems, 1(2n03):237-254, June 1998.

[JM96] D.B Johnson and D. A. Maltz. Dynamic source routing in ad hoc wireless networks, volume 353, chapter 5, pages 153-181. Kluwer Academic Publishers, Boston, 1996.

[KEOUO04] Gökhan Korkmaz, Eylem Ekici, Füsun Özgüner, and Ümit Özgüner. Urban multi-hop broadcast protocol for inter-vehicle communication systems. In Proceedings of the 1st ACM International Workshop on Vehicular ad hoc networks, pages 76-85, Philadelphia, PA, USA, Oct. 2004.

[KO07] Shahab Kamali and Jaroslav Opatrny. POSANT: A position based ant colony routing algorithm for mobile ad-hoc networks. In Proceeding of 3rd International Conference on Wireless and Mobile Communications, page 21, Guadeloupe, French Caribbean, March 2007. 
[KSLB11] Jagadeesh Kakarla, S Siva Sathya, B Govinda Laxmiand, and Ramesh Babu B. A survey on routing protocols and its issues in VANET. International Journal of Computer Applications, 28(4):38-44, Aug. 2011.

[L11] Ingo Ltkebohle. NS-2.35 source code. http://nsnam.isi.edu/nsnam/ index.php/User_Information, 2011.

[Lab08] Houda Labiod. Wireless Ad Hoc and Sensor Networks. John Wiley \& Sons, Inc., New York, USA, 1 edition, Nov. 2008.

[LG97] Chunhung Richard Lin and Mario Gerla. Adaptive clustering for mobile wireless networks. IEEE Journal on Selected Areas in Communications, 15(7):1265-1275, Sep. 1997.

$\left[\mathrm{LHT}^{+}\right] \quad$ Christian Lochert, Hannes Hartenstein, Jing Tian, Holger Fuessler, Dagmar Hermann, and Martin Mauve. A routing strategy for vehicular ad hoc networks in city environments. In Proceedings of the IEEE Intelligent Vehicles Symposium, pages 156-161, June.

[LLHG08] Kevin C. Lee, Michael Le, Jrme Hrri, and Mario Gerla. LOUVRE: Landmark overlays for urban vehicular routing environments. In Proceedings of 68th IEEE Conference on Vehicular Technology, pages 1-5, Calgary, Canada, Sept. 2008.

[LM07] Ilias Leontiadis and Cecilia Mascolo. GeOpps: Geographical opportunistic routing for vehicular networks. In Proceedings of IEEE International 
Symposium on a World of Wireless, Mobile and Multimedia Networks, pages 1-6, Helsinki, Finland, June 2007.

[LNM04] Xiaoyan Li, Thu D. Nguyen, and Richard P. Martin. Using adaptive range control to maximize 1-hop broadcast coverage in dense wireless networks. In Proceedings of 1 st Annual IEEE Communications Society Conference on Sensor and Ad Hoc Communications and Networks, pages 397-405, Santa Clara, Oct. 2004.

[MCCM08] Francisco J. Martinez, Juan-Carlos Cano, Carlos T. Calafate, and Pietro Manzoni. CityMob: A mobility model pattern generator for VANETs. In Proceedings of IEEE International Conference on Communications Workshops, pages 370-374, Beijing, China, May 2008.

[MD01] Mahesh K. Marina and Samir R. Das. On-demand multipath distance vector routing in ad hoc networks. In Proceedings of 9th IEEE International Conference on Network Protocols, pages 14-23, California, USA, Nov. 2001.

[MLF07] Hamid Menouar, Massimiliano Lenardi, and Fethi Filali. Improving proactive routing in VANETs with the MOPR movement prediction framework. In Proceedings of 7th International Conference on Intelligent Transport Systems Telecommunications, pages 1-6, Sophia Antipolis, France, June 2007.

[MLS05] Christian Maihöfer, Tim Leinmüller, and Elmar Schoch. Abiding geocast: time-stable geocast for ad hoc networks. In Proceedings of the 
2nd ACM International Workshop on Vehicular ad hoc networks, pages 20-29, Cologne, Germany, Sep. 2005.

[Mor09] Moritz Killat and Hannes Hartenstein. An empirical model for probability of packet reception in vehicular ad hoc networks. EURASIP Journal on Wireless Communications and Networking, pages 1-12, Jan. 2009.

[MP92] Michel Mouly and Marie-Bernadette Pautet. The GSM System for Mobile Communications. Telecom Publishing, 1992.

[MPGW06] Atulya Mahajan, Niranjan Potnis, Kartik Gopalan, and An-I A. Wang. Urban mobility models for vanets. In Proceedings of 2nd Workshop on next generation wireless networks, Bangalore, India, Dec. 2006.

[NAG04] Vinod Namboodiri, Manish Agarwal, and Lixin Gao. A study on the feasibility of mobile gateways for vehicular ad-hoc networks. In Proceedings of the 1st ACM International Workshop on Vehicular ad hoc networks, pages 66-75, Philadelphia, PA, USA, Oct. 2004.

[OTT08] E. Osagie, Parimala Thulasiraman, and Ruppa K. Thulasiram. PACONET: improved ant colony optimization routing algorithm for mobile ad hoc networks. In Proceedings of the 22nd International Conference on Advanced Information Networking and Applications, pages 204-211, Okinawa, Japan, 2008.

[PGC00] Guangyu Pei, Mario Gerla, and Tsu-Wei Chen. Fisheye state routing: a routing scheme for ad hoc wireless networks. In Proceedings of IEEE In- 
ternational Conference on Communications, pages 70-74, New Orleans, USA, June 2000 .

[PR99] C. Perkins and E.M. Royer. Ad-hoc on-demand distance vector routing. In Proceedings of 2nd IEEE Conference on Mobile Computing Systems and Applications, pages 90-100, New Orleans, LA, USA, February 1999.

[PSR09] Sunita Prasad, Y.P Singh, and C.S Rai. Swarm based intelligent routing for MANETs. International Journal of Recent Trends in Engineering, 1(1):153-158, May 2009.

[Rap01] Theodore Rappaport. Wireless Communications: Principles and Practice. Prentice Hall PTR, Upper Saddle River, NJ, USA, 2nd edition, Dec. 2001.

[RRC07] Lorenzo Rubio, Juan Reig, and Narcis Cardona. Evaluation of Nakagami fading behaviour based on measurements in urban scenarios. $A E U$ International Journal of Electronics and Communications, 61(2):135138, Feb. 2007.

[RRS10] B. Ramakrishnan, Dr. R. S. Rajesh, and R. S. Shaji. Performance analysis of 802.11 and 802.11p in cluster based simple highway model. Computer Science and Information Technologies, 1(5):420-426, 2010.

[SASS10] G. Samara, W.A.H. Al-Salihy, and R. Sures. Security issues and challenges of vehicular ad hoc networks (VANET). In Proceedings of 4 th 
International Conference on New Trends in Information Science and Service Science, pages 393-398, Gyeongju, South Korea, May 2010.

[SBHR96] Ruud Schoonderwoerd, Janet L. Bruten, Owen E. Holland, and Leon J. M. Rothkrantz. Ant-based load balancing in telecommunications networks. Adaptive Behavior, 5(2):169-207, Sep. 1996.

[SFL $\left.{ }^{+} 00\right]$ Min-Te Sun, Wu-Chi Feng, Ten-Hwang Lai, K. Yamada, H. Okada, and K. Fujimura. GPS-based message broadcasting for inter-vehicle communication. In Proceedings of International Conference on Parallel Processing, pages 279-286, Toronto, Canada, Aug. 2000.

[SJ04] Amit Kumar Saha and David B. Johnson. Modeling mobility for vehicular ad-hoc networks. In Proceedings of the 1st ACM International Workshop on Vehicular ad hoc networks, pages 91-92, Philadelphia, PA, USA, Oct. 2004.

[Ste05] Illya Stepanov. CanuMobisim. http://canu.informatik. uni-stuttgart.de/mobisim/, 2005.

[SYK06] Weihua Sun, Hirozumi Yamaguchi, and Shinji Kusumoto. GVGrid: A qos routing protocol for vehicular ad hoc networks. In Proceedings of 14th IEEE International Workshop on Quality of Service, pages 130139, New Haven, Connecticut, USA, June 2006.

$\left[\mathrm{TJM}^{+} 04\right]$ Vikas Taliwal, Daniel Jiang, Heiko Mangold, Chi Chen, and Raja Sengupta. Empirical determination of channel characteristics for DSRC 
vehicle-to-vehicle communication. In Proceedings of the 1st ACM International Workshop on Vehicular Ad Hoc Networks, page 88, Philadelphia, PA, USA, Oct. 2004.

[Val07] Valery Naumov and T.R.Gross. Connectivity-aware routing (CAR) in vehicular ad-hoc networks. In Proceedings of 26th IEEE International Conference on Computer Communications, pages 1919-1927, Anchorage, Alaska, May 2007.

[Wat10] Mohamed Watfa. Advances in Vehicular Ad-Hoc Networks: Developments and Challenges. IGI Global, Hershey, Pennsylvania, May 2010.

[WOTT09] Jianping Wang, Eseosa Osagie, Parimala Thulasiraman, and Ruppa K. Thulasiram. HOPNET: A hybrid ant colony optimization routing algorithm for mobile ad hoc network. Ad Hoc Networks, 7(4):690-705, 2009.

[WW11] Jing Wu and Yuhao Wang. Performance study of multi-path in VANETs and their impact on routing protocols. Wireless Engineering and Technology, 2(3):125-129, July 2011.

[WWM07] Matthias Wellens, Burkhard Westphal, and Petri Mhnen. Performance evaluation of IEEE 802.11-based WLANs in vehicular scenarios. In Proceedings of 65th IEEE Conference on Vehicular Technology Conference, pages 1167-1171, Dublin, Ireland, April 2007.

[XL08] Sun Xi and Xia-Miao Li. Study of the feasibility of VANET and its 
routing protocols. In Proceedings of 4 th International Conference on Wireless Communications, Networking and Mobile Computing, pages 1-4, Dalian, China, Oct. 2008.

[XSYA12] Weidong Xiang, Dan Shan, Jiaqi Yuan, and S. Addepalli. A full functional wireless access for vehicular environments (WAVE) prototype upon the IEEE 802.11p standard for vehicular communications and networks. In Proceedings of IEEE Consumer Communications and Networking Conference, pages 58-59, Las Vegas, NV, USA, Jan. 2012.

[YKT03] Zhenqiang Ye, Srikanth V. Krishnamurthy, and Satish K. Tripathi. A framework for reliable routing in mobile ad hoc networks. In Proceedings of the 22nd IEEE International Conference on Computer Communications), pages 270-280, San Francisco, USA, Mar. 2003.

[YRB10] Gongjun Yan, Danda B. Rawat, and Bhed Bahadur Bista. Provisioning vehicular ad hoc networks with quality of service. In Proceedings of 5th International Conference on Broadband, Wireless Computing, Communication and Applications, pages 102-107, Fukuoka, Japan, Nov. 2010.

[YW08] Bhaskar Krishnamachari Konstantinos Psounis Yi Wang, Akram Ahmed. IEEE 802.11p performance evaluation and protocol enhancement. In Proceedings of the 2008 IEEE International Conference on Vehicular Electronics and Safety, pages 22-24, Columbus, OH, USA, Sep. 2008.

[ZC08] Jing Zhao and Guohong Cao. VADD: Vehicle-assisted data delivery in 
vehicular ad hoc networks. IEEE Transactions on Vehicular Technology, 57(3):1910-1922, May 2008. 